\title{
VARIATIONAL ANALYSIS AND FULL STABILITY OF OPTIMAL SOLUTIONS TO CONSTRAINED AND MINIMAX PROBLEMS ${ }^{1}$
}

\author{
BORIS S. MORDUKHOVICH ${ }^{2}$ and M. EBRAHIM SARABI ${ }^{3}$ \\ Dedicated to Enzo Mitidieri in honor of his 60th birthday
}

\begin{abstract}
The main goal of this paper is to develop applications of advanced tools of first-order and second-order variational analysis and generalized differentiation to the fundamental notion of full stability of local minimizers of general classes of constrained optimization and minimax problems. In particular, we derive second-order characterizations of full stability and investigate its relationships with other notions of stability for parameterized conic programs and minimax problems. Furthermore, the developed variational approach allows us to largely unify and provide new self-contained proofs of some quite recent results in this direction for problems of constrained optimization with $\mathcal{C}^{2}$-smooth data.
\end{abstract}

\section{Introduction}

The notion of full stability of local minimizers in the general extended-real-valued format of unconstrained optimization was introduced by Levy, Poliquin and Rockafellar [17]; see Section 3 for more details. This notion, as well as the previous one of tilt stability [35], has drawn strong attention (especially during the recent years) of many experts on nonlinear analysis, optimization, variational inequalities, and control of partial differential equations, etc.; see [2, $6,7,8,14,18,23,24,25,26,27,28,30,31,32]$. The aforementioned publications contain various second-order characterizations of full and tilt stability in both finite-dimensional and infinite-dimensional spaces together with their applications to particular classes of optimization and control problems. Appropriate tools of second-order variational analysis and generalized differentiation play a crucial role in the obtained characterizations and subsequent applications.

The present paper continues these lines of developments in two major directions. On one hand, we establish new characterizations of full stability and its relationships with other stability notion as well as new applications to problems of conic and minimax optimization. On the other hand, we provide new, self-contained, and simplified proofs of some quite recent results on full stability obtained by different and more involved devices.

The rest of the paper is organized as follows. Section 2 briefly overviews the basic constructions of generalized differentiation in variational analysis widely used in the formulations and proofs of the main results. Section 3 addresses a general class of constrained optimization problems covering those in conic programming, establishes new properties of fully stable minimizers, and provides new proofs of major second-order characterizations of fully stable minimizers under reducibility and partial nondegeneracy conditions. In particular, the developed approach allows us to describe the framework of canonical perturbations, where full stability is equivalent to tilt stability under an appropriate parametric reduction.

Section 4 concerns relationships between full stability of local minimizers for general constrained optimization problems with $\mathcal{C}^{2}$-smooth data and Lipschitzian single-valued localizations of solution maps to the corresponding KKT (Karush-Kuhn-Tucker) systems. The obtained results with self-contained proofs ensure, in particular, the equivalence between full stability of

\footnotetext{
${ }^{1}$ This research was supported by the National Science Foundation under grant DMS-1007132.

${ }^{2}$ Department of Mathematics, Wayne State University, Detroit, MI 48202, USA and King Fahd University of Petroleum and Minerals, Dhahran, Saudi Arabia (boris@math.wayne.edu).

${ }^{3}$ Department of Mathematics, Wayne State University, Detroit, MI 48202 (ebrahim.sarabi@wayne.edu).
} 
local minimizers and Robinson's strong regularity [36] of the associated generalized equations as well as strong Lipschitz stability of stationary points with respect to $\mathcal{C}^{2}$-smooth perturbations.

Section 5 applies the obtained results on full stability and some other tools of variational analysis to calculate graphical derivatives of the normal cone mappings generated by nonconvex feasible solution sets to parameterized problems of constrained optimization written in the framework of conic programming. As a by-product of the developed approach, we establish useful Lipschitzian properties of such moving sets and derive verifiable formulas for calculating the graphical derivative of the corresponding normal cone mapping.

Section 6 is devoted to the study of full stability in unconstrained minimax problems that has never been done in the literature. Based on general characterizations of full stability in nonsmooth problems of composition optimization as well as on specific features of maximum functions, we obtain complete characterizations of fully stable optimal solutions in minimax problems entirely via their initial data by using two alternative approaches. The first proof is based on reducing the minimax problem under consideration to the framework of extended nonlinear programming and applies the corresponding characterizations of full stability obtained in our paper with Rockafellar [32]. The other approach involves exact calculations of the secondorder subdifferential for maximum functions based on the recent development by Emich and Henrion [9] and also presented below in somewhat different forms.

Throughout the paper we use the standard notation and terminology of variational analysis; cf. the books $[22,39]$.

\section{Tools of Generalized Differentiation}

Here we briefly recall some basic concepts of generalized differentiation [22, 39] widely exploited in what follows. Unless otherwise stated, all the sets $\Omega \subset \mathbb{R}^{n}$ under consideration are nonempty and locally closed and the extended-real-valued functions $\varphi: \mathbb{R}^{n} \rightarrow \overline{\mathbb{R}}:=(-\infty, \infty]$ are lower semicontinuous (l.s.c.) around the reference points.

Given $\Omega \subset \mathbb{R}^{n}$, the prenormal cone (known also as the regular or Fréchet normal cone) to $\Omega$ at $\bar{x} \in \Omega$ is defined by classical upper limit 'limsup' as

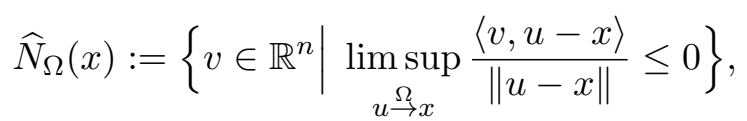

where the symbol ' $u \stackrel{\Omega}{\rightarrow} x$ ' indicates that $u \rightarrow x$ with $u \in \Omega$. The normal cone to $\Omega$ at $\bar{x} \in \Omega$ (known also as the basic/limiting/Mordukhovich normal cone) is

$$
N_{\Omega}(\bar{x})=\operatorname{Limsup}_{x \rightarrow \bar{x}} \widehat{N}_{\Omega}(x),
$$

where, given a set-valued mapping $F: \mathbb{R}^{n} \rightrightarrows \mathbb{R}^{m}$, the symbol

$$
\operatorname{Limsup}_{x \rightarrow \bar{x}} F(x):=\left\{y \in \mathbb{R}^{m} \mid \exists x_{k} \rightarrow \bar{x}, y_{k} \rightarrow y \text { with } y_{k} \in F\left(x_{k}\right), k=1,2, \ldots\right\}
$$

stands for the Painlevé-Kuratowski outer limit of $F$ as $x \rightarrow \bar{x}$. The normal cone (2.2) is often nonconvex, while its prenormal counterpart (2.1) is convex and satisfies the duality relationship

$$
\widehat{N}_{\Omega}(x)=T_{\Omega}(x)^{*}:=\left\{v \in \mathbb{R}^{n} \mid\langle v, w\rangle \leq 0 \text { for all } w \in T_{\Omega}(x)\right\}
$$

with the (Bouligand-Severi) tangent/contingent cone $T_{\Omega}(x)$ to $\Omega$ at $x \in \Omega$ defined by

$$
T_{\Omega}(x):=\left\{w \in \mathbb{R}^{n} \mid \exists x_{k} \stackrel{\Omega}{\rightarrow} x, \alpha_{k} \geq 0 \text { with } \alpha_{k}\left(x_{k}-x\right) \rightarrow w \text { as } k \rightarrow \infty\right\} .
$$


If $\Omega$ is convex, both cones (2.1) and (2.2) reduce to the classical normal cone of convex analysis.

For a function $\varphi: \mathbb{R}^{n} \rightarrow \overline{\mathbb{R}}$ finite at $\bar{x}$, the (first-order) subdifferential of $\varphi$ at $\bar{x}$ is defined, via the epigraph epi $\varphi:=\left\{(x, \alpha) \in \mathbb{R}^{n+1} \mid \alpha \geq \varphi(x)\right\}$, by

$$
\partial \varphi(\bar{x}):=\left\{v \in \mathbb{R}^{n} \mid(v,-1) \in N_{\operatorname{epi} \varphi}(\bar{x}, \varphi(\bar{x}))\right\} .
$$

Similarly the singular/horizon subdifferential of $\varphi$ at $\bar{x}$ is defined geometrically by

$$
\partial^{\infty} \varphi(\bar{x}):=\left\{v \in \mathbb{R}^{n} \mid(v, 0) \in N_{\operatorname{epi} \varphi}(\bar{x}, \varphi(\bar{x}))\right\} .
$$

It is well known that for any function $\varphi: \mathbb{R}^{n} \rightarrow \overline{\mathbb{R}}$ l.s.c. around $\bar{x}$, we have $\partial^{\infty} \varphi(\bar{x})=\{0\}$ if and only if $\varphi$ is locally Lipschitzian around $\bar{x}$. It is easy to see that $N_{\Omega}(\bar{x})=\partial \delta_{\Omega}(\bar{x})=\partial^{\infty} \delta_{\Omega}(\bar{x})$ for any $\bar{x} \in \Omega$ via the indicator function $\delta_{\Omega}(x)$ of $\Omega$ equal 0 for $x \in \Omega$ and $\infty$ otherwise.

Consider further a mapping $F: \mathbb{R}^{n} \rightrightarrows \mathbb{R}^{m}$ and define the coderivative $D^{*} F(\bar{x}, \bar{y}): \mathbb{R}^{m} \rightrightarrows \mathbb{R}^{n}$ [20] of $F$ at $(\bar{x}, \bar{y}) \in \operatorname{gph} F:=\left\{\left(x, y \in \mathbb{R}^{n} \times \mathbb{R}^{m} \mid y \in F(x)\right\}\right.$ by

$$
D^{*} F(\bar{x}, \bar{y})(v):=\left\{u \in \mathbb{R}^{n} \mid(u,-v) \in N_{\operatorname{gph} F}(\bar{x}, \bar{y})\right\}, \quad v \in \mathbb{R}^{m} .
$$

The graphical derivative $D F(\bar{x}, \bar{y}): \mathbb{R}^{n} \rightrightarrows \mathbb{R}^{m}$ of $F$ at $(\bar{x}, \bar{y}) \in \operatorname{gph} F$ is defined via $(2.3)$ by

$$
D F(\bar{x}, \bar{y})(u):=\left\{v \in \mathbb{R}^{m} \mid(u, v) \in T_{\operatorname{gph} F}(\bar{x}, \bar{y})\right\}, \quad u \in \mathbb{R}^{n} .
$$

If $F: \mathbb{R}^{n} \rightarrow \mathbb{R}^{m}$ is single-valued, we drop $\bar{y}$ in the notations (2.6) and (2.7). The smoothness of $F$ around $\bar{x}$ in the latter case yields the representations

$$
D^{*} F(\bar{x})(v)=\left\{\nabla F(\bar{x})^{*} v\right\} \text { and } D F(\bar{x})(u)=\{\nabla F(\bar{x}) u\}
$$

for all $u \in \mathbb{R}^{n}$ and $v \in \mathbb{R}^{m}$, respectively, where the symbol '*' in the first equality stands for the matrix transposition, i.e., signifies the adjoint derivative operator.

Finally, recall the construction [21] of the second-order subdifferential (or generalized Hessian) of $\varphi$ at $\bar{x}$ relative to $\bar{y} \in \partial \varphi(\bar{x})$ defined by

$$
\partial^{2} \varphi(\bar{x}, \bar{y})(u):=\left(D^{*} \partial \varphi\right)(\bar{x}, \bar{y})(u), \quad u \in \mathbb{R}^{n},
$$

via the coderivative (2.6) of the first-order subdifferential mapping (2.4). If $\varphi \in \mathcal{C}^{2}$ around $\bar{x}$, i.e., twice continuously differentiable on a neighborhood of this point, we have

$$
\partial^{2} \varphi(\bar{x})(u)=\left\{\nabla^{2} \varphi(\bar{x}) u\right\} \text { for all } u \in \mathbb{R}^{n},
$$

where $\nabla^{2} \varphi(\bar{x})$ denotes the (symmetric) Hessian matrix of $\varphi$ at $\bar{x}$.

Note that, in contrast to (2.1), (2.3), and (2.7), the nonconvex dual-space constructions (2.2), (2.4), (2.5), (2.6), and (2.8) are robust and enjoy comprehensive calculus rules based on extremal/variational principles of variational analysis; see $[22,31,39]$ and the references therein.

\section{Second-Order Characterizations of Full Stability}

This section addresses the following constrained optimization problem:

$$
\text { minimize } \varphi_{0}(x) \text { subject to } \Phi(x):=\left(\varphi_{1}(x), \ldots, \varphi_{m}(x)\right) \in \Theta \text {, }
$$

where all the functions $\varphi_{i}: \mathbb{R}^{n} \rightarrow \mathbb{R}$ for $i=0, \ldots, m$ are $\mathcal{C}^{2}$-smooth around the reference points, and where $\Theta \subset \mathbb{R}^{m}$ is a closed convex set in $\mathbb{R}^{m}$. Problems of this type belong to conic 
programming provided that $\Theta$ is a subcone of $\mathbb{R}^{m}$. Note that classical nonlinear programs (NLPs) with $s$ inequality and $m-s$ equality constraints correspond to (3.1) for $\Theta=\mathbb{R}_{-}^{s} \times\{0\}^{m-s}$.

To define full stability of local minimizers in (3.1) by reducing it to the extended unconstrained format of [17], consider the two-parametric version of (3.1) written as

$$
\mathcal{P}(w, v): \quad \text { minimize } \varphi_{0}(x, w)+\delta_{\Theta}(\Phi(x, w))-\langle v, x\rangle \text { over } x \in \mathbb{R}^{n}
$$

with the basic parameter $w \in \mathbb{R}^{d}$ and the tilt parameter $v \in \mathbb{R}^{n}$ under the the same $\mathcal{C}^{2}$-smooth assumptions on $\varphi_{0}$ and $\Phi$ with respect to both variables. Let

$$
\varphi(x, w):=\varphi_{0}(x, w)+\delta_{\Theta}(\Phi(x, w)) \text { with } x \in \mathbb{R}^{n}, w \in \mathbb{R}^{d}
$$

and fix in what follows a triple $(\bar{x}, \bar{w}, \bar{v})$ such that $\Phi(\bar{x}, \bar{w}) \in \Theta$ and $\bar{v} \in \partial_{x} \varphi(\bar{x}, \bar{w})$. Given a number $\gamma>0$, consider the (local) optimal value function

$$
m_{\gamma}(w, v):=\inf _{\|x-\bar{x}\| \leq \gamma}\{\varphi(x, w)-\langle v, x\rangle\}, \quad(w, v) \in \mathbb{R}^{d} \times \mathbb{R}^{n},
$$

and the parametric family of optimal solution sets in (3.2) defined by

$$
M_{\gamma}(w, v):=\operatorname{argmin}_{\|x-\bar{x}\| \leq \gamma}\{\varphi(x, w)-\langle v, x\rangle\}, \quad(w, v) \in \mathbb{R}^{d} \times \mathbb{R}^{n},
$$

with the convention that argmin: $=\emptyset$ when the expression under minimization is $\infty$. In this terms, $\bar{x}$ is a locally optimal solution to $\mathcal{P}(\bar{w}, \bar{v})$ if $\bar{x} \in M_{\gamma}(\bar{w}, \bar{v})$ for some $\gamma>0$ sufficiently small.

Definition 3.1 (full stability). A point $\bar{x}$ is a FULLY STABLE locally optimal solution to problem $\mathcal{P}(\bar{w}, \bar{v})$ if there exist a number $\gamma>0$ and neighborhoods $W$ of $\bar{w}$ and $V$ of $\bar{v}$ such that the mapping $(w, v) \mapsto M_{\gamma}(w, v)$ is single-valued and Lipschitz continuous with $M_{\gamma}(\bar{w}, \bar{v})=\bar{x}$ and the function $(w, v) \mapsto m_{\gamma}(w, v)$ is likewise Lipschitz continuous on $W \times V$.

This concept of full stability in the general extended-real-valued framework of $\varphi: \mathbb{R}^{n} \times \mathbb{R}^{d} \rightarrow \overline{\mathbb{R}}$ was introduced in [17]. The notion of tilt stability (the absence of the basic parameter $w$ in (3.2) and (3.3) in which case the Lipschitz continuity of (3.4) is automatic) appeared a bit earlier in [35]. Let us show that Definition 3.1 can be equivalently reformulated in a weaker form. In the case of tilt stability it can be deduced from [14, Corollary 4.7] proved in a different way.

Recall that $F: \mathbb{R}^{n} \rightrightarrows \mathbb{R}^{m}$ is Lipschitz-like (or has the Aubin property) around $(\bar{x}, \bar{y}) \in \operatorname{gph} F$ with modulus $l \geq 0$ if there are neighborhoods $U$ of $\bar{x}$ and $V$ of $\bar{y}$ such that

$$
F(x) \cap V \subset F(u)+l\|x-u\| \mathbb{B} \text { whenever } x, u \in U .
$$

Recall also that $F: \mathbb{R}^{n} \rightrightarrows \mathbb{R}^{n}$ is monotone provided that

$$
\left\langle y_{2}-y_{1}, x_{2}-x_{1}\right\rangle \geq 0 \text { for all }\left(x_{1}, y_{1}\right),\left(x_{2}, y_{2}\right) \in \operatorname{gph} F \text {. }
$$

Theorem 3.2 (equivalent description of full stability). Definition 3.1 can be equivalently reformulated by replacing the single-valuedness and Lipschitz continuity of the solution map $M_{\gamma}$ around $(\bar{w}, \bar{v})$ by the Lipschitz-like property of this mapping around $(\bar{w}, \bar{v}, \bar{x})$.

Proof. By the Lipschitz-like property of $M_{\gamma}$ around $(\bar{w}, \bar{v}, \bar{x})$, find a neighborhood triple $(W, V, U)$ for $(\bar{w}, \bar{v}, \bar{x})$ such that (3.6) holds for $M_{\gamma}$ in this notation. Fix $w \in W$ and define

$$
\varphi_{w}(\cdot)=\varphi(\cdot, w), \quad \widetilde{\varphi}_{w}:=\varphi_{w}+\delta_{\mathbb{B}_{\gamma}(\bar{x})}, \quad g_{w}:=\widetilde{\varphi}_{w}^{*},
$$


where the latter stands for the conjugate of $\widetilde{\varphi}_{w}$. Thus $g_{w}$ is convex being expressed as

$$
g_{w}(v)=\max _{x \in \mathbb{B}_{\gamma}(\bar{x})}\left\{\langle v, x\rangle-\varphi_{w}(x)\right\}, \quad v \in \mathbb{R}^{n} .
$$

Considering further the set-valued mapping $T_{w}: \mathbb{R}^{n} \rightrightarrows \mathbb{R}^{n}$ defined by $T_{w}(v):=M_{\gamma}(w, v)$ on $V$ and $T_{w}(v):=\emptyset$ otherwise, we claim that it is monotone. Indeed, pick $x_{i} \in T_{w}\left(v_{i}\right)$ with $v_{i} \in V$ as $i=1,2$ and get from (3.7) the relationships

$$
\begin{aligned}
\left\langle x_{1}-x_{2}, v_{1}-v_{2}\right\rangle & =\left\langle x_{1}, v_{1}\right\rangle-\left\langle x_{2}, v_{1}\right\rangle-\left\langle x_{1}, v_{2}\right\rangle+\left\langle x_{2}, v_{2}\right\rangle \\
& =\left[g_{w}\left(v_{1}\right)-\left\langle x_{2}, v_{1}\right\rangle+\varphi_{w}\left(x_{2}\right)\right]+\left[g_{w}\left(v_{2}\right)-\left\langle x_{1}, v_{2}\right\rangle+\varphi_{w}\left(x_{1}\right)\right] \geq 0
\end{aligned}
$$

which verify the claimed monotonicity of $T_{\bar{w}}$. Since the mapping $v \mapsto T_{\bar{w}}(v)=M_{\gamma}(\bar{w}, v)$ is Lipschitz-like (i.e., surely lower semicontinuous) around $(\bar{v}, \bar{x})$, the classical Kenderov theorem [13] tells us that $T_{\bar{w}}(\bar{v})=\{\bar{x}\}$, and hence $M_{\gamma}(\bar{w}, \bar{v})=\{\bar{x}\}$. The same arguments work for any $(w, v) \in W \times V$ justifying therefore the single-valuedness of $M_{\gamma}$ on $W \times V$.

Recall that the (partial) Robinson constraint qualification (abbr. RCQ) holds for (3.1) at $(\bar{x}, \bar{w})$ with $\Phi(\bar{x}, \bar{w}) \in \Theta$ if we have

$$
N_{\Theta}(\Phi(\bar{x}, \bar{w})) \cap \operatorname{ker} \nabla_{x} \Phi(\bar{x}, \bar{w})^{*}=\{0\} .
$$

Corollary 3.3 (full stability under RCQ). Assume that the mapping $(w, v) \mapsto M_{\gamma}(w, v)$ in (3.5) is Lipschitz-like around $(\bar{x}, \bar{w}, \bar{v}) \in \operatorname{gph} M_{\gamma}$ and that RCQ (3.8) holds at $(\bar{x}, \bar{w})$. Then $\bar{x}$ is a fully stable locally optimal solution to problem $\mathcal{P}(\bar{w}, \bar{v})$.

Proof. Employing Theorem 3.2, it remains to show that the value function (3.4) is Lipschitz continuous around $(\bar{w}, \bar{v})$. The latter is a consequence of [17, Propositions 2.2,3.5] and the fact that $M_{\gamma}(\bar{w}, \bar{v})=\{\bar{x}\}$, which is also due to Theorem 3.2.

For the reader's convenience we formulate now the result of [32, Theorem 5.1] in the setting of (3.1) that provides a characterization of fully stable local minimizers for the unperturbed problem $\mathcal{P}(w, v)$ in $(3.2)$ under the full rank condition. In what follows we are going to essentially relax this condition, which this theorem is used in our further considerations.

Theorem 3.4 (characterizing fully stable local minimizers under full rank condition). Let $\bar{x}$ be a feasible solution to $\mathcal{P}(\bar{w}, \bar{v})$ in (3.1) with the fixed parameter pair $(\bar{w}, \bar{v})$ as above under the validity of the full rank/surjectivity condition

$$
\operatorname{rank} \nabla_{x} \Phi(\bar{x}, \bar{w})=m .
$$

Let $\bar{z}:=\Phi(\bar{x}, \bar{w})$, and let $\bar{\lambda} \in \mathbb{R}^{m}$ be the unique vector satisfying the KKT system

$$
\nabla_{x} \Phi(\bar{x}, \bar{w})^{*} \bar{\lambda}+\nabla_{x} \varphi_{0}(\bar{x}, \bar{w})=\bar{v} \text { and } \bar{\lambda} \in N_{\Theta}(\bar{z}) .
$$

Then $\bar{x}$ is a fully stable local minimizer for $\mathcal{P}(\bar{w}, \bar{v})$ if and only if we have the implication

$$
[(p, q) \in \mathcal{T}(\bar{x}, \bar{w}, \bar{v})(u), u \neq 0] \Longrightarrow\langle p, u\rangle>0
$$

for the set-valued mapping $\mathcal{T}(\bar{x}, \bar{w}, \bar{v}): \mathbb{R}^{n} \rightrightarrows \mathbb{R}^{n} \times \mathbb{R}^{d}$ defined by

$$
\begin{aligned}
\mathcal{T}(\bar{x}, \bar{w}, \bar{v})(u) & :=\left(\nabla_{x x}^{2} \varphi_{0}(\bar{x}, \bar{w}) u, \nabla_{x w}^{2} \varphi_{0}(\bar{x}, \bar{w}) u\right)+\left(\nabla_{x x}^{2}\langle\bar{\lambda}, \Phi\rangle(\bar{x}, \bar{w}) u, \nabla_{x w}^{2}\langle\bar{\lambda}, \Phi\rangle(\bar{x}, \bar{w}) u\right) \\
& +\left(\nabla_{x} \Phi(\bar{x}, \bar{w}), \nabla_{w} \Phi(\bar{x}, \bar{w})\right)^{*} \partial^{2} \delta_{\Theta}(\bar{z}, \bar{\lambda})\left(\nabla_{x} \Phi(\bar{x}, \bar{w}) u\right), \quad u \in \mathbb{R}^{n} .
\end{aligned}
$$


Observe that in the setting of Theorem 3.4 we have

$$
[(0, q) \in \mathcal{T}(\bar{x}, \bar{w}, \bar{v})(0)] \Longrightarrow q=0
$$

Indeed the latter implication is equivalent to

$$
\left[\nabla_{x} \Phi(\bar{x}, \bar{w})^{*} p=0, \nabla_{w} \Phi(\bar{x}, \bar{w})^{*} p=q, p \in \partial^{2} \delta_{\Theta}(\bar{z}, \bar{y})(0)\right] \Longrightarrow q=0
$$

which holds automatically under the full rank assumption (3.9).

Note that for NLPs the full rank condition (3.9) reduces to the classical linear independence constraint qualification (LICQ). For the general constrained problem (3.1) this condition does not depend on the underlying set $\Theta$ and thus readily calls for a possible improvement.

We now recall two conditions from [2, Definition 3.135], widely recognized in the framework of (3.1), and extend Theorem 3.4 to this general setting by reducing it to the full rank case (3.9).

(RC) The closed convex set $\Theta \subset \mathbb{R}^{m}$ is $\mathcal{C}^{2}$-reducible at $\bar{z}=\Phi(\bar{x}, \bar{w}) \in \Theta$ to the closed convex set $\Xi \subset \mathbb{R}^{p}$ if there is a neighborhood $U$ of $\bar{z}$ and a $\mathcal{C}^{2}$-smooth mapping $h: U \rightarrow \mathbb{R}^{p}$ with the surjective derivative operator $\nabla h(\bar{z}): \mathbb{R}^{m} \rightarrow \mathbb{R}^{p}$ such that $\delta_{\Theta}(z)=\delta_{\Xi}(h(z))$ for all $z \in U$ and the tangent cone $T_{\Xi}(h(\bar{z}))$ is pointed. If this holds for all $z \in \Theta$, then we say that $\Theta$ is $\mathcal{C}^{2}$-reducible to $\Xi$. Without loss of generality we assume that $h(\bar{z})=0$.

(ND) We say that $(\bar{x}, \bar{w})$ in (RC) is a partially nondegenerate point for $\Phi$ with respect to $\Theta$ if

$$
\nabla_{x} \Phi(\bar{x}, \bar{w}) \mathbb{R}^{n}+\operatorname{lin}\left\{T_{\Theta}(\bar{z})\right\}=\mathbb{R}^{m},
$$

where $\operatorname{lin}\left\{T_{\Theta}(\bar{z})\right\}$ signifies the largest linear subspace contained in $T_{\Theta}(\bar{z})$.

It is well known that the reducibility condition (RC) holds for many important classes of problems in constrained optimization. This includes the cases when $\Theta$ is a polyhedral set, a Lorentz (second-order, ice-cream) cone, and the cone of positive semidefinite matrices; see, e.g., [2]. The nondegeneracy condition (ND) is more restrictive. It follows from [2, Proposition 4.73] that (3.13) can be equivalently reformulated in the dual form

$$
\operatorname{span}\left\{N_{\Theta}(\bar{z})\right\} \cap \operatorname{ker} \nabla_{x} \Phi(\bar{x}, \bar{w})^{*}=\{0\}
$$

which shows that it reduces to LICQ for the case of NLPs, being however essentially less restrictive than the latter even for polyhedral sets $\Theta$ as in [32, Example 6.9].

To proceed further, impose (RC) and deduce from it that the original constraint $\Phi(x, w) \in \Theta$ in (3.1) is locally equivalent to $h(\Phi(x, w)) \in \Xi$. This allows us to conclude that problem $\mathcal{P}(w, v)$ in $(3.2)$ locally around $(\bar{x}, \bar{w})$ amounts to the reduced problem as follows:

$$
\mathcal{P}_{r}(w, v) \quad\left\{\begin{array}{l}
\text { minimize } \varphi_{0}(x, w)-\langle v, x\rangle \text { subject to } x \in \mathbb{R}^{n} \\
\Psi(x, w):=h(\Phi(x, w)) \in \Xi
\end{array}\right.
$$

which can be equivalently rewritten as

$$
\text { minimize } \varphi_{0}(x, w)+\delta_{\Xi}(\Psi(x, w))-\langle v, x\rangle \text { over } x \in \mathbb{R}^{n} .
$$

The next result shows that, while full stability issues for (3.2) and (3.15) are equivalent, we have the full rank condition for the reduced problem under the validity of (ND) for the original one. 
Proposition 3.5 (full stability and nondegeneracy in the original and reduced problems). Let $\bar{x}$ be a feasible solution to $\mathcal{P}(\bar{w}, \bar{v})$ in (3.2) along the fixed parameter pair $(\bar{w}, \bar{v})$, and let condition (RC) hold. Then $\bar{x}$ is a fully stable locally optimal solution to $\mathcal{P}(\bar{w}, \bar{v})$ if and only if it is a fully stable locally optimal solution to the reduced problem $\mathcal{P}_{r}(\bar{w}, \bar{v})$. Furthermore, the validity in addition of (ND) for $(\bar{x}, \bar{w})$ implies the surjectivity of $\nabla_{x} \Psi(\bar{x}, \bar{w})$ for $\Psi$ in (3.15).

Proof. The claimed equivalence follows directly from representation (3.16) of the reduced problem with $\Psi$ from (3.15) and the definition of full stability. To prove the second part of the proposition, assume (ND) for $(\bar{x}, \bar{w})$ in $(3.2)$ and get by (RC) and [2, Proposition 4.73] that

$$
\operatorname{lin}\left\{T_{\Theta}(\bar{z})\right\}=T_{\Omega}(\bar{z}) \text { with } \Omega:=\{z \in U \mid h(z)=0\},
$$

where $U$ is given in (RC). Taking into account the representation of the tangent cone to $\Omega$ from [39, Example 6.8], the nondegeneracy condition (3.13) reduces now to

$$
\nabla_{x} \Phi(\bar{x}, \bar{w}) \mathbb{R}^{n}+\operatorname{ker} \nabla h(\bar{z})=\mathbb{R}^{m}
$$

Using this together with the surjectivity of $\nabla h(\bar{z})$ we get by the classical chain rule that

$$
\nabla_{x} \Psi(\bar{x}, \bar{w}) \mathbb{R}^{n}=\nabla h(\bar{z}) \nabla_{x} \Phi(\bar{x}, \bar{w}) \mathbb{R}^{n}=\nabla h(\bar{z})\left(\nabla_{x} \Phi(\bar{x}, \bar{w}) \mathbb{R}^{n}+\operatorname{ker} \nabla h(\bar{z})\right)=\nabla h(\bar{z}) \mathbb{R}^{m}=\mathbb{R}^{p},
$$

which justifies the surjectivity of $\nabla_{x} \Psi(\bar{x}, \bar{w})$ and completes the proof of the proposition.

Observe further from the standard subdifferential sum and chain rules [22, 39] applied to (3.3) under (3.8) that the stationary condition $\bar{v} \in \partial_{x} \varphi(\bar{x}, \bar{w})$ for (3.2) yields

$$
\bar{v} \in \nabla_{x} \varphi_{0}(\bar{x}, \bar{w})+\nabla_{x} \Phi(\bar{x}, \bar{w})^{*} N_{\Theta}(\Phi(\bar{x}, \bar{w})) .
$$

This leads us to the KKT system (3.10), which can be equivalently rewritten as

$$
\bar{v}=\nabla_{x} L(\bar{x}, \bar{w}, \bar{\lambda}), \quad \bar{\lambda} \in N_{\Theta}(\Phi(\bar{x}, \bar{w}))
$$

via the Lagrangian $L(x, w, \lambda):=\varphi_{0}(x, w)+\langle\lambda, \Phi(x, w)\rangle$ for (3.2). It is well known (see, e.g., [2, Proposition 4.75]) that (3.18) admits the unique Lagrange multiplier under the validity of (ND).

Similarly we define the KKT system associated with reduced problem (3.15) by

$$
\bar{v}=\nabla_{x} L_{r}(\bar{x}, \bar{w}, \bar{\mu}), \quad \bar{\mu} \in N_{\Xi}(\Psi(\bar{x}, \bar{w})),
$$

where $L_{r}$ is the Lagrangian for (3.15) given by $L_{r}(x, w, \mu):=\varphi_{0}(x, w)+\langle\mu, \Psi(x, w)\rangle$. This system surely has the unique solution due to the full rank result of Proposition 3.5.

The next important result provides a second-order subdifferential characterization of full stability for $\mathcal{P}(\bar{w}, \bar{v})$ at nondegenerate solutions by reducing it to the full rank setting of Theorem 3.4. Our proof is essentially different from the original one given recently in [26, Theorem 5.6], which is based on the unform quadratic growth characterization of Robinson's strong regularity of the associated KKT system/generalized equation obtained in [2, Theorem 5.24].

Theorem 3.6 (second-order subdifferential characterization of full stability of nondegenerate solutions in constrained optimization). Let $\bar{x}$ be a feasible solution to the unperturbed problem $\mathcal{P}(\bar{w}, \bar{v})$ in (3.2) with some $\bar{w} \in \mathbb{R}^{d}$ and $\bar{v}$ from (3.17). Assume further (RC) and (ND) hold, and let $\bar{\lambda}$ be the unique vector satisfying (3.18). Then $\bar{x}$ is a fully stable local minimizer of $\mathcal{P}(\bar{w}, \bar{v})$ if and only if we have

$$
\left\langle u, \nabla_{x x}^{2} L(\bar{x}, \bar{w}, \bar{\lambda}) u\right\rangle+\left\langle q, \nabla_{x} \Phi(\bar{x}, \bar{w}) u\right\rangle>0
$$

for all $q \in \partial^{2} \delta_{\Theta}(\bar{z}, \bar{\lambda})\left(\nabla_{x} \Phi(\bar{x}, \bar{w}) u\right)$ with $u \neq 0$. 
Proof. Starting with verifying the "only if" part, let $\bar{x}$ be a fully stable local minimizer for $\mathcal{P}(\bar{w}, \bar{v})$ and hence for the reduced problem $\mathcal{P}_{r}(\bar{w}, \bar{v})$ in $(3.15)$ by the first part of Proposition 3.5. The second part of this proposition ensures that $\nabla_{x} \Psi(\bar{x}, \bar{w})$ is surjective under the assumptions made. Then Theorem 3.4 tells us that implication (3.11) holds with replacing $\mathcal{T}(\bar{x}, \bar{w}, \bar{v})$ by the set-valued mapping $\widehat{\mathcal{T}}_{r}(\bar{x}, \bar{w}, \bar{v}): \mathbb{R}^{n} \rightrightarrows \mathbb{R}^{n} \times \mathbb{R}^{d}$ defined by

$$
\begin{aligned}
\widehat{\mathcal{T}}_{r}(\bar{x}, \bar{w}, \bar{v})(u): & =\left(\nabla_{x x}^{2} \varphi_{0}(\bar{x}, \bar{w}) u, \nabla_{x w}^{2} \varphi_{0}(\bar{x}, \bar{w}) u\right)+\left(\nabla_{x x}^{2}\langle\bar{\mu}, \Psi\rangle(\bar{x}, \bar{w}) u, \nabla_{x w}^{2}\langle\bar{\mu}, \Phi\rangle(\bar{x}, \bar{w}) u\right) \\
& +\left(\nabla_{x} \Psi(\bar{x}, \bar{w}), \nabla_{w} \Psi(\bar{x}, \bar{w})\right)^{*} \partial^{2} \delta_{\Xi}(\bar{z}, \bar{\mu})\left(\nabla_{x} \Psi(\bar{x}, \bar{w}) u\right), \quad u \in \mathbb{R}^{n},
\end{aligned}
$$

where $\bar{\mu}$ is the unique solution of the reduced KKT system (3.19). Using now the second-order chain rule from [31, Theorem 3.1] under the full rank assumption leads us to

$$
\widehat{\mathcal{T}}_{r}(\bar{x}, \bar{w}, \bar{v})(u)=\left(\nabla_{x x}^{2} \varphi_{0}(\bar{x}, \bar{w}) u, \nabla_{x w}^{2} \varphi_{0}(\bar{x}, \bar{w}) u\right)+D^{*} \partial_{x}\left(\delta_{\Xi} \circ \Psi\right)(\bar{x}, \bar{w}, \bar{v})(u) .
$$

On the other hand, it follows from (RC) that $\left(\delta_{\Xi} \circ \Psi\right)(x, w)=\left(\delta_{\Theta} \circ \Phi\right)(x, w)$ for all $(x, w)$ around $(\bar{x}, \bar{w})$. Using this together with $(3.21)$, we get

$$
\widehat{\mathcal{T}}_{r}(\bar{x}, \bar{w}, \bar{v})(u)=\left(\nabla_{x x}^{2} \varphi_{0}(\bar{x}, \bar{w}) u, \nabla_{x w}^{2} \varphi_{0}(\bar{x}, \bar{w}) u\right)+D^{*} \partial_{x}\left(\delta_{\Theta} \circ \Phi\right)(\bar{x}, \bar{w}, \bar{v})(u) .
$$

Finally, the result of [34, Theorem 7] held under (ND) ensures that

$$
\widehat{\mathcal{T}}_{r}(\bar{x}, \bar{w}, \bar{v})(u)=\mathcal{T}(\bar{x}, \bar{w}, \bar{v})(u), \quad u \in \mathbb{R}^{n},
$$

which justifies together with (3.11) that condition (3.20) is satisfied.

To verify now the "if" part, assume the validity of (3.20) and deduces from (3.22) that it also holds for $\widehat{\mathcal{T}}_{r}(\bar{x}, \bar{w}, \bar{v})$; hence we get implication (3.11) for the latter mapping. By the surjectivity of $\nabla_{x} \Psi(\bar{x}, \bar{w})$ it follows from Theorem 3.4 that $\bar{x}$ is a fully stable local minimizer of the reduced problem $\widehat{\mathcal{P}}(\bar{w}, \bar{v})$ and thus for the original problem $\mathcal{P}(\bar{w}, \bar{v})$ by Proposition 3.5.

Remark 3.7 (enhanced second-order condition). An important point hidden in the proof of Theorem 3.6 and used below is that assumptions (RC) and (ND) ensure the validity of implication (3.12), which was established previously under the full rank condition. To elaborate it more, take $(0, q) \in \mathcal{T}(\bar{x}, \bar{w}, \bar{v})(0)$ and observe from the discussion above that it yields

$$
\nabla_{x} \Phi(\bar{x}, \bar{w})^{*} p=0 \text { and } \nabla_{w} \Phi(\bar{x}, \bar{w})^{*} p=q \text { with some } p \in \partial^{2} \delta_{\Theta}(\bar{z}, \bar{\lambda})(0) .
$$

Employing (RC), we get $\Theta \cap U=h^{-1}(\Xi) \cap U$ in the notation therein. It follows from [22, Theorem 1.17] by the surjectivity of $\nabla_{x} \Psi(\bar{x}, \bar{w})$ for $\Psi=h \circ \Phi$ that $N_{\Theta}(\bar{z})=\nabla h(\bar{z})^{*} N_{\Xi}(\Psi(\bar{x}, \bar{w}))$. Appealing now to [31, Theorem 3.1] gives us $d \in \partial^{2} \delta_{\Xi}(\Psi(\bar{x}, \bar{w}), \bar{\mu})(0)$ such that $p=\nabla h(\bar{z})^{*} d$, where $\bar{\mu} \in N_{\Xi}(\Psi(\bar{x}, \bar{w}))$ is the unique solution to the reduced KKT system (3.19) satisfying $\bar{\lambda}=\nabla h(\bar{z})^{*} \bar{\mu}$. Thus $\nabla_{x} \Psi(\bar{x}, \bar{w})^{*} d=0$ due to (3.23), which shows that $d=0$ and hence $p=0$. Substitution $p=0$ into (3.23) justifies (3.12).

Recall that the validity of implication (3.12) under assumptions (RC) and ( ND) was first proved in [32, Theorem 6.6] for mathematical programs with polyhedral constraint (i.e., when $\Theta$ in (3.1) is as polyhedral set) and then in [30, Lemma 4.5] for second-order cone programs when $\Theta$ stands for the Lorentz second-order/ice-cream cone.

As a consequence of the discussions in Remark 3.7, we show next that that the validity of (ND) under (RC) implies the following second-order qualification condition (SOQC)

$$
\partial^{2} \delta_{\Theta}(\bar{z}, \bar{\lambda})(0) \cap \operatorname{ker} \nabla_{x} \Phi(\bar{x}, \bar{w})^{*}=\{0\}
$$


from [31], where $\bar{\lambda}$ is the unique solution of KKT system (3.18) at the given triple $(\bar{x}, \bar{w}, \bar{v})$. Note that the converse implication holds when $\Theta$ is either a polyhedral convex set [28, Proposition 6.1], or the Lorentz second-order cone [30, Theorem 3.6], or the SDP cone $\mathcal{S}_{+}^{m}$ (this can be derived from [3]), while in general it still remains an open question.

Corollary 3.8 (second-order qualification condition under nondegeneracy). Let $\bar{\lambda}$ be the unique vector satisfying (3.18) for the triple $(\bar{x}, \bar{w}, \bar{v})$ from Theorem 3.6 , and let conditions (RC) and (ND) be fulfilled. Then SOCQ (3.24) holds.

Proof. Take $p \in \partial^{2} \delta_{\Theta}(\bar{z}, \bar{\lambda})(0)$ with $\nabla_{x} \Phi(\bar{x}, \bar{w})^{*} p=0$ and find from the discussions in Theorem 3.6 and Remark 3.7 a vector $d \in \partial^{2} \delta_{\Xi}(\Psi(\bar{x}, \bar{w}), \bar{\mu})(0)$ such that $p=\nabla h(\bar{z})^{*} d$ in the notation above. This gives us $\nabla_{x} \Psi(\bar{x}, \bar{w})^{*} d=0$ and hence $d=0$ by the surjectivity of $\nabla_{x} \Psi(\bar{x}, \bar{w})$. It shows that $p=0$ and completes the proof.

The next consequence of Theorem 3.6 opens a technical gate for obtaining the main result of Section 4 given in Theorem 4.2. To proceed, consider the following canonically perturbed version of problem (3.1) with parametric pairs $\left(v_{1}, v_{2}\right) \in \mathbb{R}^{n} \times \mathbb{R}^{m}$ :

$$
\widetilde{\mathcal{P}}_{\bar{w}}\left(v_{1}, v_{2}\right) \quad\left\{\begin{array}{l}
\text { minimize } \varphi_{0}(x, \bar{w})-\left\langle v_{1}, x\right\rangle \text { subject to } x \in \mathbb{R}^{n} \\
\Phi(x, \bar{w})+v_{2} \in \Theta .
\end{array}\right.
$$

Corollary 3.9 (full stability with respect to canonical perturbations). Let $\bar{x}$ be $a$ feasible solution to the unperturbed problem $\mathcal{P}(\bar{w}, \bar{v})$ in (3.2) with some $\bar{w} \in \mathbb{R}^{d}$ and $\bar{v}$ from (3.17), and assumptions (RC) and (ND) be satisfied. Then $\bar{x}$ is a fully stable local minimizer of $\mathcal{P}(\bar{w}, \bar{v})$ if and only if it is a fully stable local minimizer of $\widetilde{\mathcal{P}}_{\bar{w}}(\bar{v}, 0)$.

Proof. We can easily to see that the nondegeneracy condition (ND) for $\mathcal{P}(\bar{w}, \bar{v})$ at $\bar{x}$ is equivalent to the validity of this condition for $\widetilde{\mathcal{P}}_{\bar{w}}(\bar{v}, 0)$. It follows from Theorem 3.6 that the full stability of the local minimizer $\bar{x}$ in both problems $\mathcal{P}(\bar{w}, \bar{v})$ and $\widetilde{\mathcal{P}}_{\bar{w}}(\bar{v}, 0)$ amounts to the validity of the same second-order condition (3.20). This justifies the claimed equivalence.

Looking at the problem $\widetilde{\mathcal{P}}_{\bar{w}}(\bar{v}, 0)$ in Corollary 3.9 , observe that it corresponds to just the tilt perturbation of the original problem (3.2) with the fixed basic parameter $w=\bar{w}$. The latter problem can be written as $\mathcal{P}_{\bar{w}}(v)$. Thus we have the following consequence of Corollary 3.9 about the relationship between full and tilt stability under the assumptions made.

Corollary 3.10 (reduction of full stability to tilt stability at nondegenerate solutions.) Consider the setting of Corollary 3.9. Then the full stability of the local minimizer $\bar{x}$ for the original problem $\mathcal{P}(\bar{w}, \bar{v})$ is equivalent to its tilt stability in problem $\mathcal{P}_{\bar{w}}(\bar{v})$.

Proof. It follows from the discussion above that both stability notions are characterized by the same second-order condition (3.20) under the (RC) and (ND) assumptions made.

\section{Relationships of Full Stability with Other Stability Notions}

This section addresses relationships between full stability of our basic problem $\mathcal{P}(\bar{w}, \bar{v})$ in $(3.2)$ and other well-recognized stability notions in constrained optimization and associated variational systems. We develop a largely self-contained approach to such relationships based on the reduction procedure of Section 3, which allows us to establish new equivalences and also to provide new proofs of some recently discovered results in this direction.

We first present a rather simple description of full stability in $\mathcal{P}(\bar{w}, \bar{v})$ via a Lipschitzian single-valued localization of the parameterized collection of stationary points therein. Recall 
that a set-valued mapping $F: \mathbb{R}^{n} \rightrightarrows \mathbb{R}^{m}$ admits a single-valued graphical localization around $(\bar{x}, \bar{y}) \in \operatorname{gph} F$ provided that there exist neighborhoods $U$ of $\bar{x}$ and $V$ of $\bar{y}$ together with a single-valued mapping $f: U \rightarrow V$ such that $\operatorname{gph} F \cap(U \times V)=\operatorname{gph} f$.

Proposition 4.1 (equivalence between full stability of $\mathcal{P}(\bar{w}, \bar{v})$ and Lipschitzian localization of parameterized stationary points). Let $\bar{x}$ be a feasible solution to the unperturbed problem $\mathcal{P}(\bar{w}, \bar{v})$ in (3.2) with some $\bar{w} \in \mathbb{R}^{d}$ and $\bar{v}$ from (3.17), and let RCQ (3.8) hold. Then $\bar{x}$ is a fully stable locally optimal solution to problem $\mathcal{P}(\bar{w}, \bar{v})$ if and only if $\bar{x} \in M_{\gamma}(\bar{w}, \bar{v})$ for some $\gamma>0$ and the set-valued mapping

$$
S(w, v):=\left\{x \in \mathbb{R}^{n} \mid v \in \nabla_{x} \varphi_{0}(x, w)+\nabla_{x} \Phi(x, w)^{*} N_{\Theta}(\Phi(x, w))\right\}
$$

admits a Lipschitzian single-valued graphical localization around $(\bar{w}, \bar{v}, \bar{x})$.

Proof. Applying the corresponding characterization of full stability in the general unconstrained format of [32, Theorem 3.4] with the extended-real-valued function $\varphi$ from (3.3), we conclude as in the proof of Proposition 3.5 above that the stationary condition $\bar{v} \in \partial_{x} \varphi(\bar{x}, \bar{w})$ is equivalent to (3.17). It remains to observe that the basic constraint qualification imposed on [32, Theorem 3.4] holds due to the assumed RCQ by [17, Proposition 2.2].

The Lipschitzian property of the set-valued mapping $S$ in (4.1) is known from [32, Definition 3.3] as partial strong metric regularity. This notion is a partial version of strong metric regularity of $\partial_{x} \varphi$ at $(\bar{x}, \bar{w}, \bar{v})$ introduced in [5] as an abstract version of Robinson's strong regularity in the framework of generalized equations [36].

Unless otherwise stated, in the rest of this section we take $\bar{v}=0$ in the KKT system (3.18) without less of generality. Consider the generalized equation (GE)

$$
\left[\begin{array}{l}
v \\
0
\end{array}\right] \in\left[\begin{array}{c}
\nabla_{x} L(x, w, \lambda) \\
-\Phi(x, w)
\end{array}\right]+\left[\begin{array}{c}
0 \\
N_{\Theta}^{-1}(\lambda)
\end{array}\right]
$$

which is indeed the KKT system for problem $\mathcal{P}(w, v)$ in $(3.2)$. Let $(\bar{x}, \bar{\lambda})$ be a solution to $(4.2)$ with $(w, v)=(\bar{w}, 0)$ and define the partial linearization of $(4.2)$ at $(\bar{x}, \bar{\lambda})$ by

$$
\left[\begin{array}{l}
v_{1} \\
v_{2}
\end{array}\right] \in\left[\begin{array}{c}
\nabla_{x x}^{2} L(\bar{x}, \bar{w}, \bar{\lambda})(x-\bar{x})+\nabla_{x} \Phi(\bar{x}, \bar{w})^{*}(\lambda-\bar{\lambda}) \\
-\Phi(\bar{x}, \bar{w})-\nabla_{x} \Phi(\bar{x}, \bar{w})(x-\bar{x})
\end{array}\right]+\left[\begin{array}{c}
0 \\
N_{\Theta}^{-1}(\lambda)
\end{array}\right] .
$$

Recall [36] that $(\bar{x}, \bar{\lambda})$ is a strongly regular solution to the KKT system (4.2) if the solution map to (4.3) has a Lipschitz continuous single-valued localization around $(0,0) \in \mathbb{R}^{n} \times \mathbb{R}^{m}$.

Theorem 4.2 (full stability of $\mathcal{P}(\bar{w}, \bar{v})$ and local single-valuedness and Lipschitz continuity of solution maps to basic and reduced KKT systems). Let $\bar{x}$ be a feasible solution to the unperturbed problem $\mathcal{P}(\bar{w}, \bar{v})$ in $(3.2)$ with some $\bar{w} \in \mathbb{R}^{d}$ and $\bar{v}=0$ from (3.17) under the validity of the reducibility (RC) and $R C Q$ conditions. The following are equivalent:

(i) $\bar{x}$ is a fully stable locally optimal solution to $\mathcal{P}(\bar{w}, \bar{v})$ satisfying (ND).

(ii) $\bar{x} \in M_{\gamma}(\bar{w}, \bar{v})$ for some $\gamma>0$ and the solution map $S_{K K T}^{r}:(w, v) \mapsto(x, \mu)$ for the reduced KKT system (3.19) is single-valued and Lipschitz continuous around $(\bar{w}, \bar{v}, \bar{x}, \bar{\mu})$.

(iii) $\bar{x} \in M_{\gamma}(\bar{w}, \bar{v})$ for some $\gamma>0$ and the solution map $S_{K K T}:(w, v) \mapsto(x, \lambda)$ for the KKT system (3.18) is single-valued and Lipschitz continuous around $(\bar{w}, \bar{v}, \bar{x}, \bar{\lambda})$.

(iv) $\bar{x} \in M_{\gamma}(\bar{w}, \bar{v})$ for some $\gamma>0$ and $(\bar{x}, \bar{\lambda})$ is a strongly regular solution to (4.2). 
Proof. To verify implication $(\mathbf{i}) \Longrightarrow$ (ii), we get from (i) and the first part of Proposition 3.5 that $\bar{x}$ is a fully stable locally optimal solution to the reduced problem (3.15). The local singlevaluedness of the solution map $S_{K K T}^{r}:(w, v) \mapsto(x, \mu)$ for the reduced KKT system (3.19) in (ii) was established above as a consequence of the imposed (RC) and (ND) assumptions ensuring the full rank condition for $\nabla_{x} \Psi(\bar{x}, \bar{w})$ by the second part of Proposition 3.5.

Next we verify that the mapping $S_{K K T}^{r}:(w, v) \mapsto(x, \mu)$ is Lipschitz continuous around $(\bar{w}, \bar{v}, \bar{x}, \bar{\mu})$. Note that the Lipschitz continuity of $(w, v) \mapsto x_{w v}$ comes directly from the full stability of $\bar{x}$ in (3.15). To justify this property for the mapping $(w, v) \mapsto \mu_{w v}$, pick $w_{1}, w_{2} \in W$ and $v_{1}, v_{2} \in V$ and then find $\mu_{w_{i} v_{i}} \in N_{\Xi}\left(c_{i}\right)$ with $c_{i}:=\Psi\left(x_{w_{i} v_{i}}, w_{i}\right)$ for $i=1,2$ satisfying

$$
\left\{\begin{array}{l}
v_{2}=\nabla_{x} \varphi_{0}\left(x_{w_{2} v_{2}}, w_{2}\right)+\nabla_{x} \Psi\left(x_{w_{2} v_{2}}, w_{2}\right)^{*} \mu_{w_{2} v_{2}} \\
v_{1}=\nabla_{x} \varphi_{0}\left(x_{w_{1} v_{1}}, w_{1}\right)+\nabla_{x} \Psi\left(x_{w_{1} v_{1}}, w_{1}\right)^{*} \mu_{w_{1} v_{1}}
\end{array}\right.
$$

It shows therefore that the validity of the following equality

$$
\begin{aligned}
\nabla_{x} \Psi\left(x_{w_{2} v_{2}}, w_{2}\right)^{*}\left(\mu_{w_{2} v_{2}}-\mu_{w_{1} v_{1}}\right) & =\left(\nabla_{x} \Psi\left(x_{w_{1} v_{1}}, w_{1}\right)-\nabla_{x} \Psi\left(x_{w_{2} v_{2}}, w_{2}\right)\right)^{*} \mu_{w_{1} v_{1}} \\
& +\nabla_{x} \varphi_{0}\left(x_{w_{1} v_{1}}, w_{1}\right)-\nabla_{x} \varphi_{0}\left(x_{w_{2} v_{2}}, w_{2}\right)+v_{2}-v_{1}
\end{aligned}
$$

By shrinking the neighborhoods $W$ and $V$ if necessary, we can always assume the surjectivity of $\nabla_{x} \Psi\left(x_{w_{i} v_{i}}, w_{i}\right)$ due to this property of $\nabla_{x} \Psi(\bar{x}, \bar{w})$. Thus it follows from the standard surjectivity result of [22, Lemma 1.18] that for any $(w, v) \in W \times V$ there is $\kappa_{w v}>0$ such that

$$
\left\|\nabla_{x} \Psi\left(x_{w_{2} v_{2}}, w_{2}\right)^{*}\left(\mu_{w_{2} v_{2}}-\mu_{w_{1} v_{1}}\right)\right\| \geq \kappa_{w_{2} v_{2}}\left\|\mu_{w_{2} v_{2}}-\mu_{w_{1} v_{1}}\right\| \geq \kappa\left\|\mu_{w_{2} v_{2}}-\mu_{w_{1} v_{1}}\right\|,
$$

where $\kappa:=\inf \left\{\kappa_{w v} \mid(w, v) \in W \times V\right\}$. Furthermore, it is easy to conclude from the surjectivity of $\nabla_{x} \Psi(\bar{x}, \bar{w})$ that $\kappa>0$ and that there is $\rho<\infty$ such that $\left\|\mu_{w v}\right\| \leq \rho$ for all $(w, v) \in W \times V$. Denoting by $\ell>0$ is a common Lipschitz constant for the mappings $\nabla_{x} \varphi_{0}, \nabla_{x} \Phi, \nabla h$, $\Phi$, and $(w, v) \mapsto x_{w v}$ on $W \times V$, we derive from (4.4) and (4.5) the estimates

$$
\begin{aligned}
\left\|\mu_{w_{2} v_{2}}-\mu_{w_{1} v_{1}}\right\| & \leq \frac{1}{\kappa}\left(\left\|\nabla_{x} \Psi\left(x_{w_{1} v_{1}}, w_{1}\right)-\nabla_{x} \Psi\left(x_{w_{2} v_{2}}, w_{2}\right)\right\| \cdot\left\|\mu_{w_{1} v_{1}}\right\|\right. \\
& \left.+\left\|\nabla_{x} \varphi_{0}\left(x_{w_{1} v_{1}}, w_{1}\right)-\nabla_{x} \varphi_{0}\left(x_{w_{2} v_{2}}, w_{2}\right)\right\|+\left\|v_{2}-v_{1}\right\|\right) \\
& \leq \frac{\gamma}{\kappa}\left[\rho \ell^{2}\left(\left\|x_{w_{2} v_{2}}-x_{w_{1} v_{1}}\right\|+\left\|w_{2}-w_{1}\right\|\right)\right. \\
& \left.+\ell\left(\left\|x_{w_{2} v_{2}}-x_{w_{1} v_{1}}\right\|+\left\|w_{2}-w_{1}\right\|\right)+\left\|v_{2}-v_{1}\right\|\right] .
\end{aligned}
$$

which imply the local Lipschitz continuity of $(w, v) \mapsto \mu_{w v}$ and thus justify (ii).

Next we show that assuming the local Lipschitz continuity of $S_{K K T}^{r}:(w, v) \mapsto(x, \mu)$ around $(\bar{w}, \bar{v}, \bar{x}, \bar{\mu})$ in (ii) implies this property for $S_{K K T}:(w, v) \mapsto(x, \lambda)$ around $(\bar{w}, \bar{v}, \bar{x}, \bar{\lambda})$ with $\bar{\lambda}:=$ $\nabla h(\bar{x})^{*} \bar{\mu}$ and $\bar{z}:=\Phi(\bar{x}, \bar{w})$ in (iii). Similarly to the above, it remains to verify that the mapping $(w, v) \mapsto \lambda_{w v}$ is Lipschitz continuous around $(\bar{w}, \bar{v})$. To proceed, take any $w_{i} \in W, v_{i} \in V$ and form $\lambda_{w_{i} v_{i}}:=\nabla h\left(z_{i}\right)^{*} \mu_{w_{i} v_{i}}$ with $z_{i}:=\Phi\left(x_{w_{i} v_{i}}, w_{i}\right)$ for $i=1,2$. Then we have

$$
\begin{aligned}
\left\|\lambda_{w_{2} v_{2}}-\lambda_{w_{1} v_{1}}\right\| & =\left\|\nabla h\left(z_{2}\right)^{*} \mu_{w_{2} v_{2}}-\nabla h\left(z_{1}\right)^{*} \mu_{w_{1} v_{1}}\right\| \\
& \leq\left\|\nabla h\left(z_{2}\right)^{*}\right\| \cdot\left\|\mu_{w_{2} v_{2}}-\mu_{w_{1} v_{1}}\right\|+\left\|\nabla h\left(z_{2}\right)-\nabla h\left(z_{1}\right)\right\| \cdot\left\|\mu_{w_{1} v_{1}}\right\| \\
& \leq \tau\left\|\mu_{w_{2} v_{2}}-\mu_{w_{1} v_{1}}\right\|+\rho \ell^{2}\left(\left\|x_{w_{2} v_{2}}-x_{w_{1} v_{1}}\right\|+\left\|w_{2}-w_{1}\right\|\right)
\end{aligned}
$$

in the notation above, where $\tau>0$ is an upper bound of $\left\|\nabla h(z)^{*}\right\|$ for all $z$ sufficiently close to $\bar{z}$. This justifies the claimed local Lipschitz continuity of $S_{K K T}$ and thus verifies (iii).

Our next implication to prove is (iii) $\Longrightarrow(\mathbf{i})$. Taking into account Proposition 4.1 and the form of the KKT system (3.18), it remains to check that (iii) ensures the validity of (ND). This 
kind of relationships has been well understood in optimization theory (see, e.g., [2]); we present a complete proof in our setting for the reader's convenience.

Arguing by contradiction, suppose that (ND) in the equivalent form (3.13) does not hold and thus find $0 \neq \vartheta \in \mathbb{R}^{m}$ so that $\nabla_{x} \Phi(\bar{x}, \bar{w})^{*} \vartheta=0$ and that $\vartheta \in \operatorname{span}\left\{N_{\Theta}(\bar{z})\right\}$ with $\bar{z}=\Phi(\bar{x}, \bar{w})$. By (iii) we have $S_{K K T}(\bar{w}, \bar{v})=\{(\bar{x}, \bar{\lambda})\}$ for some $\bar{\lambda} \in \mathbb{R}^{m}$. If $\bar{\lambda} \in \operatorname{ri} N_{\Theta}(\bar{z})$, with "ri" standing for the relative interior of a convex set, then $\bar{\lambda}+t \vartheta \in N_{\Theta}(\bar{z})$ for any small $t>0$. Indeed, it is easy to see that $\operatorname{span}\left\{N_{\Theta}(\bar{z})\right\}=\operatorname{aff}\left\{N_{\Theta}(\bar{z})\right\}$ and hence $\bar{\lambda}+t \vartheta \in$ aff $\left\{N_{\Theta}(\bar{z})\right\}$ when $t>0$ is sufficiently small. Employing this, we get $(\bar{x}, \bar{\lambda}+t \vartheta) \in S_{K K T}(\bar{w}, \bar{v})$, which contradicts the the aforementioned uniqueness of Lagrange multipliers in (3.18) and so justifies (ND) in this case. In the remaining case of $\bar{\lambda} \notin$ ri $N_{\Theta}(\bar{z})$, pick $\xi \in$ ri $N_{\Theta}(\bar{z}) \neq \emptyset$ and get from the wellknown result of convex analysis (see, e.g., [39, Proposition 2.40]) that $\bar{\lambda}+t(\xi-\bar{\lambda}) \in \operatorname{ri} N_{\Theta}(\bar{z})$ for any $t \in(0,1)$. Putting $v_{t}=t \nabla_{x} \Phi(\bar{x}, \bar{w})^{*}(\xi-\bar{\lambda})$ for $t>0$ sufficiently small, we obtain that $(\bar{x}, \bar{\lambda}+t(\xi-\bar{\lambda})) \in S_{K K T}\left(\bar{w}, v_{t}\right)$. Since $\bar{\lambda}+t(\xi-\bar{\lambda}) \in$ ri $N_{\Theta}(\bar{z})$, it again justifies (ND) by the arguments above and thus confirms the validity of assertion (i).

To verify now implication $(\mathbf{i}) \Longrightarrow(\mathbf{i v})$, take $\bar{x}$ from $(\mathbf{i})$ and deduce from Corollary 3.9 that $\bar{x}$ is a fully stable locally optimal solution to problem $\widetilde{\mathcal{P}}_{\bar{w}}(\bar{v}, 0)$ defined by $(3.25)$ with $\bar{v}=0$. Note that the KKT system for the parametric problem $\widetilde{\mathcal{P}}_{\bar{w}}\left(v_{1}, v_{2}\right)$ is given by

$$
\left[\begin{array}{l}
v_{1} \\
v_{2}
\end{array}\right] \in\left[\begin{array}{c}
\nabla_{x} L(x, \bar{w}, \lambda) \\
-\Phi(x, \bar{w})
\end{array}\right]+\left[\begin{array}{c}
0 \\
N_{\Theta}^{-1}(\lambda)
\end{array}\right]
$$

where $\left(v_{1}, v_{2}\right)$ varies around $(\bar{v}, 0) \in \mathbb{R}^{n} \times \mathbb{R}^{m}$. It follows from the implication $(\mathbf{i}) \Longrightarrow$ (iii) established above that the solution map $\widetilde{S}_{K K T}:\left(v_{1}, v_{2}\right) \mapsto(x, \lambda)$ for $(4.6)$ is single-valued and Lipschitz continuous around $(\bar{v}, 0)$. Observe that the generalized equation (4.3) can be treated as a (partial) linearization of the KKT system (4.6). Taking into account that (4.6) is a canonically perturbed system, we conclude that the local single-valuedness and Lipschitz continuity of its solution map is equivalent to these properties of solutions to its linearization (4.3); see, e.g., [5, Theorem 2B.10]). The latter justifies the strong regularity of the KKT system (3.18) around $(\bar{x}, \bar{\lambda})$ according to the definition above taken from [36].

To complete the proof of the theorem, it remains to show that (iv) $\Longrightarrow(\mathbf{i})$. Take $\bar{x}$ satisfying (iv) with some $\bar{\lambda}$. Then the arguments of the preceding paragraph tell us that the solution map $\widetilde{S}_{K K T}$ for the KKT system (4.6) is single-valued and Lipschitz continuous around $(\bar{v}, 0)$. Employing now in this setting the implication (iii) $\Longrightarrow(\mathbf{i})$ established above ensures that $\bar{x}$ is a fully stable locally optimal solution to problem $\widetilde{\mathcal{P}}_{\bar{w}}(\bar{v}, 0)$ satisfying (ND). Thus it is a fully stable locally optimal solution to the original problem $\mathcal{P}(\bar{w}, \bar{v})$ by Corollary 3.9.

Note that the equivalence (i) $\Longleftrightarrow$ (iv) of Theorem 4.2 has been recently proved in [26, Theorem 5.6] by using a more sophisticated device based on characterizing strong regularity in [2] via the uniform quadratic growth condition with respect to the so-called $\mathcal{C}^{2}$-smooth parametrization defined below. Furthermore, the latter growth condition has been employed in [26] to characterize yet another stability notion known as strong Lipschitzian stability. In theorem 4.3 we relate this notion to full stability by using a new approach via Theorem 3.6 and Proposition 4.1. Note that the first part of Theorem 4.3 does not impose (RC) in contrast to [26, Theorem 5.6].

To proceed, fix $\bar{w} \in \mathbb{R}^{d}$ and consider the constrained optimization problem

$$
\mathcal{P}_{\bar{w}}: \quad \text { minimize } \varphi_{0}(x, \bar{w}) \text { subject to } \Phi(x, \bar{w}) \in \Theta
$$

with the data from (3.1). We say that the pair $(\vartheta(x, u), \Upsilon(x, u))$ with $u \in \mathbb{R}^{s}$ and $\vartheta: \mathbb{R}^{n} \times \mathbb{R}^{s} \rightarrow \mathbb{R}$, $\Upsilon: \mathbb{R}^{n} \times \mathbb{R}^{s} \rightarrow \mathbb{R}^{m}$ is a $\mathcal{C}^{2}$-smooth parametrization of $\left(\varphi_{0}(x, \bar{w}), \Phi(x, \bar{w})\right)$ in $(4.7)$ at $\bar{u} \in \mathbb{R}^{s}$ if $\varphi_{0}(x, \bar{w})=\vartheta(x, \bar{u})$ and $\Phi(x, \bar{w})=\Upsilon(x, \bar{u})$ for all $x \in \mathbb{R}^{n}$, where both $\vartheta$ and $\Upsilon$ are twice 
continuously differentiable. Define the family of parametric optimization problems:

$$
\widehat{\mathcal{P}}(u) \quad\left\{\begin{array}{l}
\text { minimize } \vartheta(x, u) \text { subject to } x \in \mathbb{R}^{n}, \\
\Upsilon(x, u) \in \Theta .
\end{array}\right.
$$

We say [2, Definition 5.33] that a stationary point $\bar{x}$ of $\mathcal{P}_{\bar{w}}$ is strongly Lipschitz stable with respect to the $\mathcal{C}^{2}$-smooth parametrization $(\vartheta(x, u), \Upsilon(x, u))$ of $\left(\varphi_{0}(x, \bar{w}), \Phi(x, \bar{w})\right)$ in $(4.7)$ at $\bar{u} \in \mathbb{R}^{s}$ if there are neighborhoods $U$ of $\bar{u}$ and $X$ of $\bar{x}$ such that for any $u \in U$ each problem $\widehat{\mathcal{P}}(u)$ has the unique stationary point $x(u) \in X$ and the mapping $u \longmapsto x(u)$ is Lipschitz continuous around $\bar{u}$. If it holds for any $\mathcal{C}^{2}$-smooth parameterizations of $\left(\varphi_{0}(x, \bar{w}), \Phi(x, \bar{w})\right)$ in $(4.7)$ at $\bar{u} \in \mathbb{R}^{s}$, then $\bar{x}$ is called strongly Lipschitz stable. This notion is a Lipschitzian counterpart of the Kojima's strong stability [15], where the mapping $u \longmapsto x(u)$ is merely continuous.

Theorem 4.3 (full stability vs. strong Lipschitzian stability in constrained optimization. Let $\bar{x}$ be a Lipschitz stable locally optimal solution to problem $\mathcal{P}_{\bar{w}}$ in the framework of Proposition 4.1. Then it is a fully stable locally optimal solution to problem $\mathcal{P}(\bar{w}, \bar{v})$ with $\bar{v}=0$. The converse implication holds provided that both (RC) and (ND) conditions are satisfied.

Proof. To justify the first part of the theorem, take a Lipschitz stable locally optimal solution to (4.7). It is easy to see that $\left(\varphi_{0}(x, w)-\langle x, v\rangle, \Phi(x, w)\right)$ is a $\mathcal{C}^{2}$-smooth parametrization of $\left(\varphi_{0}(x, \bar{w}), \Phi(x, \bar{w})\right)$ in $(4.7)$ at $\bar{u}:=(\bar{w}, 0) \in \mathbb{R}^{d} \times \mathbb{R}^{n}$. Let $x(u)$ be the unique stationary point $x(u)$ for any $u=(w, v)$ close enough to $\bar{u}$ and so that the mapping $u \longmapsto x(u)$ is Lipschitz continuous around $\bar{u}$. This tells us that the set-valued mapping

$$
S(u):=\left\{x \in \mathbb{R}^{n} \mid v \in \nabla_{x} \varphi_{0}(x, w)+\nabla_{x} \Phi(x, w)^{*} N_{\Theta}(\Phi(x, w))\right\}
$$

has a Lipschitzian single-valued graphical localization around $(\bar{u}, \bar{x})$. Employing now Proposition 4.1 , we deduce that $\bar{x}$ is a fully stable locally optimal solution to problem $\mathcal{P}(\bar{w}, 0)$.

To prove the converse implication of the theorem, suppose that $\bar{x}$ is a fully stable locally optimal solution to problem $\mathcal{P}(\bar{w}, 0)$ under the validity of (RC) and (ND). By Theorem 3.6 we have the second-order characterization (3.20). Take now an arbitrary $\mathcal{C}^{2}$-smooth parametrization of $\left(\varphi_{0}(x, \bar{w}), \Phi(x, \bar{w})\right)$ in $(4.7)$ at $\bar{u} \in \mathbb{R}^{s}$. This yields the equalities $\nabla_{x} \varphi_{0}(\bar{x}, \bar{w})=\nabla_{x} \vartheta(\bar{x}, \bar{u})$, $\nabla_{x} \Phi(\bar{x}, \bar{w})=\nabla_{x} \Upsilon(\bar{x}, \bar{u})$ as well as those for the corresponding second-order derivatives. Thus we have (3.6) for problem $\widehat{\mathcal{P}}(\bar{u})$, which ensures that $\bar{x}$ is a fully stable locally optimal solution to this problem. Then it follows from Proposition 4.1 that the set-valued mapping

$$
S(u, v):=\left\{x \in \mathbb{R}^{n} \mid v \in \nabla_{x} \vartheta(x, u)+\nabla_{x} \Upsilon(x, u)^{*} N_{\Theta}(\Upsilon(x, u))\right\}
$$

admits a Lipschitzian single-valued graphical localization around $(\bar{u}, 0)$. Letting now $x(u):=$ $S(u, 0)$, we get that $x(u)$ is a stationary point for problem $\widehat{\mathcal{P}}(u)$ and that the mapping $u \longmapsto x(u)$ is locally Lipschitz continuous around $\bar{u}$. This verifies the strong Lipschitzian stability of $\bar{x}$ in (4.7) and thus completes the proof of the theorem.

\section{Graphical Derivatives of Parametric Normal Cone Mappings}

In this section we study some aspects of variational analysis and generalized differentiation for parameterized constraint systems given by

$$
\Gamma(w):=\left\{x \in \mathbb{R}^{n} \mid \Phi(x, w) \in \Theta\right\}, \quad w \in \mathbb{R}^{d},
$$


which appear in the full stability framework of Section 2. We keep the same assumptions on $\Phi$ and $\Theta$ as in Section 2 and define the normal cone mapping

$$
\Xi(x, w):=N_{\Gamma(w)}(x) \text { for } x \in \Gamma(w), w \in \mathbb{R}^{d}
$$

with $\Xi(x, w):=\emptyset$ for $x \notin \Gamma(w)$ generated by normals $(2.2)$ to the moving sets (5.1). The main goal of this section is to calculate the graphical derivative (2.7) of the mapping (5.2). To accomplish this goal and related issues, we employ the full stability results obtained above along with the other machinery of variational analysis.

Recall that a l.s.c. function $\psi: \mathbb{R}^{n} \times \mathbb{R}^{d} \rightarrow \overline{\mathbb{R}}$ is prox-regular in $x \in \mathbb{R}^{n}$ at $\bar{x}$ for $\bar{v} \in \partial_{x} \psi(\bar{x}, \bar{w})$ with compatible parameterization by $w \in \mathbb{R}^{d}$ at $\bar{w}$ if there are neighborhoods $U$ of $\bar{x}, W$ of $\bar{w}$, and $V$ of $\bar{v}$ together with numbers $\varepsilon>0$ and $\gamma \geq 0$ such that

$$
\begin{aligned}
& \psi(u, w) \geq \psi(x, w)+\langle v, u-x\rangle-\frac{\gamma}{2}\|u-x\|^{2} \text { for all } u \in U \\
& \text { when } v \in \partial_{x} \psi(x, w) \cap V, x \in U, w \in W, \psi(x, w) \leq \psi(\bar{x}, \bar{w})+\varepsilon .
\end{aligned}
$$

Consider the (Euclidean) metric projection operator $\Pi_{\Gamma(w)}: \mathbb{R}^{n} \rightrightarrows \mathbb{R}^{n}$ with some $w \in \mathbb{R}^{d}$ and observed that the condition $x \in \Gamma(w)$ yields $\Phi(x, w) \in \Theta$ and $x \in \Pi_{\Gamma(w)}(x)$.

The first lemma of this section reveals nice properties of the mapping $\Gamma$ in (5.1) and the normal cone mapping (5.2) generated by it under the validity of RCQ (3.8). The second assertion of this lemma is a general counterpart of [19, Lemma 5] proved there for NLPs under the constant rank qualification condition (CRCQ) replacing our (3.8), which in this case reduces to the (partial) Mangasarian-Fromovitz constraint qualification (MFCQ). It has been well recognized that the conditions CRCQ and MFCQ/RCQ are mutually independent.

Recall that a mapping $F: \mathbb{R}^{n} \rightrightarrows \mathbb{R}^{m}$ is inner semicontinuous at $(\bar{x}, \bar{y}) \in \operatorname{gph} F$ if for any neighborhood $U$ of $\bar{x}$ there is a neighborhood $V$ of $\bar{y}$ such that $F(x) \cap V \neq \emptyset$ for all $x \in U$.

Lemma 5.1 (single-valued localization of projections). Assume that $\Phi(\bar{x}, \bar{w}) \in \Theta$ and that $R C Q(3.8)$ holds at $(\bar{x}, \bar{w})$. Then we have the following assertions:

(i) The set-valued mapping $\Gamma$ in (5.1) is Lipschitz-like around $(\bar{w}, \bar{x}) \in \operatorname{gph} \Gamma$.

(ii) There exist numbers $\varepsilon, \nu>0$ and a neighborhood $W$ of $\bar{w}$ such that the graphical localization to $\mathbb{B}_{\varepsilon}(\bar{p}) \times W \times \mathbb{B}_{\nu}(\bar{x})$ of the set-valued mapping $(p, w) \mapsto\left(I+N_{\Gamma(w)}\right)^{-1}(p)$ is a single-valued mapping $\pi(p, w)$ that coincides with the graphical localization to $\mathbb{B}_{\varepsilon}(\bar{p}) \times W \times \mathbb{B}_{\nu}(\bar{x})$ of the setvalued mapping $(p, w) \mapsto \Pi_{\Gamma(w)}(p)$ for $\bar{p}=\bar{x}$.

Proof. To verify (i), we employ [22, Theorem 4.37(ii)] ensuring the Lipschitz-like property of $\Gamma$ around $(\bar{w}, \bar{x}) \in \operatorname{gph} \Gamma$ provided that

$$
(0, q) \in\left(\nabla_{x} \Phi(\bar{x}, \bar{w}), \nabla_{w} \Phi(\bar{x}, \bar{w})\right)^{*} N_{\Theta}(\bar{z}) \Longrightarrow q=0,
$$

which is clearly satisfied under the assumed RCQ condition (3.8). Since the Lipschitz-like property in (i) readily yields the inner semicontinuity of $\Gamma$ at $(\bar{w}, \bar{x})$, assertion (ii) of the theorem follows from [40, Theorem 3.2] provided that the indicator function $\delta_{\Gamma(w)}(x)$ is prox-regular in $x$ at $\bar{x}$ for $\bar{v}$ with compatible parameterization by $w$ at $\bar{w}$; cf. also [10, Theorem 2.3] in the nonparametric case. It is worth noting that this fact from [40] is a (Hilbert space) extension of the corresponding result from [37, Theorem 2], where $\Gamma$ is assumed to be continuous around $\bar{w}$ that may not hold in our setting.

To complete the proof, it remains to observe that the aforementioned prox-regularity of $\delta_{\Gamma(w)}(x)$ follows from [17, Proposition 2.2] due to the strong amenability of this function, which 
is a consequence of the fact that $\Gamma(w)$ is a strongly amenable set [39, Definition 10.23] for any $w$ close to $\bar{w}$ under the assumed RCQ condition; see, e.g., [39, Excercise 10.25].

The obtained Lemma 5.1(ii) allows us to associate locally the single-valued projection $\pi(p, w)$ with the projection operator $\Pi_{\Gamma(w)}(p)$ and also with the mapping $\left(I+N_{\Gamma(w)}\right)^{-1}(p)$, which we do in the rest of the section. The next result justifies the existence of the directional derivative of the projection operator and calculates its directional derivative via solutions of the generalized equation associated with (5.1). It provides a parametric extension of the recent result from [28, Lemma 3.1] derived under a rather restrictive assumption on the convexity of $\Gamma$.

To proceed, consider the parametric generalized equation

$$
0 \in x-p+N_{\Gamma(w)}(x),
$$

which can be equivalently rewritten locally around $(\bar{x}, \bar{w})$ as

$$
\left[\begin{array}{l}
0 \\
0
\end{array}\right] \in\left[\begin{array}{c}
x-p+\nabla_{x} \Phi(x, w)^{*} \lambda \\
-\Phi(x, w)
\end{array}\right]+\left[\begin{array}{c}
0 \\
N_{\Theta}^{-1}(\lambda)
\end{array}\right]
$$

For the rest of this section we assume that the convex set $\Theta$ is $\mathcal{C}^{2}$-cone reducible for all $z \in \Theta$ around $\bar{z}$, which means that the convex set $\Xi$ in definition of (RC) is a pointed cone at such points. This condition, denoted by (RCC), allows us to employ [1, Theorem 7.2] and [41, Theorem 1.1] and conclude that the projection mapping $\Pi_{\Theta}$ is directionally differentiable. We thank Héctor Ramírez and Alex Shapiro for communicating to us this observation.

Theorem 5.2 (directional differentiability of projections to moving sets). Given a vector $\bar{x} \in \Gamma(\bar{w})$ with some $\bar{w} \in \mathbb{R}^{d}$, assume that conditions (RCC) and (ND) are satisfied. Then there is a neighborhood $O$ of $(\bar{x}, \bar{w})$ such that the projection mapping $\pi(p, w)=\Pi_{\Gamma(w)}(p)$ is directionally differentiable at any $(p, w) \in O$ in every direction $\left(h_{1}, h_{2}\right) \in \mathbb{R}^{n} \times \mathbb{R}^{d}$. Furthermore, its directional derivative is calculated by $\pi^{\prime}\left((p, w) ;\left(h_{1}, h_{2}\right)\right)=k_{1}$, where $k_{1}$ is the first component of the unique solution $\left(k_{1}, k_{2}\right) \in \mathbb{R}^{n} \times \mathbb{R}^{m}$ to the system of equations

$$
\begin{aligned}
h_{1} & =\left(I+\sum_{i=1}^{m} \lambda_{i} \nabla_{x x}^{2} \Phi_{i}(x, w)\right) k_{1}+\sum_{i=1}^{m} \lambda_{i} \nabla_{x w}^{2} \Phi_{i}(x, w) h_{2}+\nabla_{x} \Phi(x, w)^{*} k_{2}, \\
0 & =\nabla_{x} \Phi(x, w) k_{1}+\nabla_{w} \Phi(x, w) h_{2}-\Pi_{\Theta}^{\prime}\left(\Phi(x, w)+\lambda ; \nabla_{x} \Phi(x, w) k_{1}+\nabla_{w} \Phi(x, w) h_{2}+k_{2}\right)
\end{aligned}
$$

with $(x, \lambda)$ being the unique solution to the generalized equation (5.4) corresponding to the parameter pair $(p, w)$ satisfying $\pi(p, w)=x$.

Proof. Observe first that $\bar{x}$ solves (5.3) with $(p, w)=(\bar{x}, \bar{w})$ since $0 \in N_{\Gamma(\bar{w})}(\bar{x})$ due to $\bar{x} \in \Gamma(\bar{w})$. Define now the parametric optimization problem $\mathcal{P}\left(p, w, v_{1}, v_{2}\right)$ by

$$
\text { minimize } \frac{1}{2}\|x-p\|^{2}-\left\langle v_{1}, x\right\rangle \text { over } \Phi(x, w)+v_{2} \in \Theta
$$

where $p, v_{1} \in \mathbb{R}^{n}$ and $\left(w, v_{2}\right) \in \mathbb{R}^{d} \times \mathbb{R}^{m}$. Our goal is to show that $\bar{x}$ is a fully stable local minimizer of problem $\mathcal{P}(\bar{p}, \bar{w}, 0,0)$ with $\bar{p}=\bar{x}$. Since assumptions (RC) and (ND) are satisfied for $(5.5)$ and since $(\bar{x}, \bar{w})$ is a feasible solution of $\mathcal{P}(\bar{p}, \bar{w}, 0,0)$, we can employ the full stability characterization from Theorem 3.6. This amounts to verifying the second-order condition (3.20), which reduces to checking that for for any $0 \neq u \in \mathbb{R}^{n}$ we have

$$
\|u\|^{2}+\left\langle\nabla_{x x}^{2} \Phi(\bar{x}, \bar{w})(u, u), \bar{\lambda}\right\rangle+\left\langle\nabla_{x} \Phi(\bar{x}, \bar{w}) u, q\right\rangle>0 \text { if } q \in \partial^{2} \delta_{\Theta}(\bar{x}, \bar{\lambda})\left(\nabla_{x} \Phi(\bar{x}, \bar{w}) u\right),
$$

where $\bar{\lambda}$ is the unique solution to system (5.4) associated with $(\bar{x}, \bar{p}, \bar{w})$. It follows that $\bar{\lambda}=0$ since $\bar{p}=\bar{x}$. Employing further [35, Theorem 2.1] tells us that $\left\langle\nabla_{x} \Phi(\bar{x}, \bar{w}) u, q\right\rangle \geq 0$ for any 
$q \in \partial^{2} \delta_{\Theta}(\bar{x}, \bar{\lambda})\left(\nabla_{x} \Phi(\bar{x}, \bar{w}) u\right)$ due to the maximal monotonicity of $N_{\Theta}$. This justifies (5.6) for any $u \neq 0$ and thus verifies that $\bar{x}$ is a fully stable local minimizer of $\mathcal{P}(\bar{p}, \bar{w}, 0,0)$. Equivalence $(\mathbf{i}) \Longleftrightarrow(\mathbf{i v})$ in Theorem 4.2 shows that the KKT system for $\mathcal{P}\left(p, w, v_{1}, v_{2}\right)$ given by

$$
\left[\begin{array}{l}
v_{1} \\
v_{2}
\end{array}\right] \in\left[\begin{array}{c}
x-p+\nabla_{x} \Phi(x, w)^{*} \lambda \\
-\Phi(x, w)
\end{array}\right]+\left[\begin{array}{c}
0 \\
N_{\Theta}^{-1}(\lambda)
\end{array}\right]
$$

is strongly regular at $(\bar{x}, \bar{\lambda})$. We claim now the slightly modified generalized equation

$$
\left[\begin{array}{l}
v_{1} \\
v_{2}
\end{array}\right] \in\left[\begin{array}{c}
x-p+\nabla_{x} \Phi(x, w)^{*}\left(\lambda+v_{2}\right) \\
-\Phi(x, w)
\end{array}\right]+\left[\begin{array}{c}
0 \\
N_{\Theta}^{-1}(\lambda)
\end{array}\right]
$$

has the same property at $(\bar{x}, \bar{\lambda})$. Indeed, it is easy to see that the partial linearization of GE $(5.7)$ at $(\bar{x}, \bar{\lambda})$ with $\left(p, w, v_{1}, v_{2}\right)=(\bar{p}, \bar{w}, 0,0)$ corresponds to the partial linearization of GE (5.8) at $(\bar{x}, \bar{\lambda})$ with $\left(p, w, v_{1}, v_{2}\right)=(\bar{p}, \bar{w}, 0,0)$. Denoting by $T$ the solution map to (5.8), we get from $[2$, Theorem 5.13] that $T$ is single-valued and Lipschitz continuous around $(\bar{p}, \bar{w}, 0,0, \bar{x}, \bar{\lambda})$. Thus there are neighborhoods $O_{1}$ of $\bar{p}, O_{2}$ of $\bar{w}, O_{3}$ of $0_{\mathbb{R}^{n}}, O_{4}$ of $0_{\mathbb{R}^{m}}, O_{5}$ of $\bar{x}$, and $O_{6}$ of $\bar{\lambda}$ such that $T\left(p, w, v_{1}, v_{2}\right) \cap\left(O_{5} \times O_{6}\right)=\left\{\sigma\left(p, w, v_{1}, v_{2}\right)\right\}$ for any $\left(p, w, v_{1}, v_{2}\right) \in O_{1} \times O_{2} \times O_{3} \times O_{4}$ with $T(\bar{p}, \bar{w}, 0,0)=(\bar{x}, \bar{\lambda})$, where $\sigma: O_{1} \times O_{2} \times O_{3} \times O_{4} \rightarrow O_{5} \times O_{6}$ is single-valued and Lipschitz continuous. Define now the mapping $\Lambda: \mathbb{R}^{n} \times \mathbb{R}^{d} \times \mathbb{R}^{n} \times \mathbb{R}^{m} \rightarrow \mathbb{R}^{n} \times \mathbb{R}^{d} \times \mathbb{R}^{n} \times \mathbb{R}^{m}$ by

$$
\Lambda(p, w, x, \lambda):=\left[\begin{array}{l}
p \\
w \\
x-p+\nabla_{x} \Phi(x, w)^{*} \lambda \\
\Phi(x, w)-\Pi_{\Theta}(\Phi(x, w)+\lambda)
\end{array}\right]
$$

Without loss of generality we suppose that $\frac{1}{2} O_{4}+\frac{1}{2} O_{5} \subset O_{5}$ and thus get

$$
\begin{gathered}
\Lambda^{-1}\left(p, w, v_{1}, v_{2}\right) \cap\left(O_{1} \times O_{2} \times O_{6} \times \frac{1}{2} O_{5}\right)=\left(p, w, \sigma_{1}\left(p, w, v_{1}, v_{2}\right), \sigma_{2}\left(p, w, v_{1}, v_{2}\right)\right) \\
\text { whenever }\left(p, w, v_{1}, v_{2}\right) \in O_{1} \times O_{2} \times O_{3} \times \frac{1}{2} O_{4},
\end{gathered}
$$

where $\sigma_{1}$ and $\sigma_{2}$ are the components of $\sigma$. This shows that $\Lambda$ is a Lipschitzian homeomorphism near $(\bar{p}, \bar{w}, \bar{x}, \bar{\lambda})$. Using this and the directional differentiability of the projection operator $\Pi_{\Theta}$ under (RCC) (this follows from [1, Theorem 7.2] as well as from [41, Theorem 1.1] due to [2, Proposition 3.136]) allows us to apply Kummer's inverse mapping theorem [16] (see also [33, Lemma 6.1]) and conclude that the single-valued and Lipschitz continuous mapping $\rho:=$ $\Lambda^{-1} \cap\left(O_{1} \times O_{2} \times O_{6} \times \frac{1}{2} O_{5}\right)$ is directionally differentiable at $\Lambda(p, w, x, \lambda) \in O_{1} \times O_{2} \times O_{3} \times \frac{1}{2} O_{4}$ with the directional derivative calculated by

$$
\rho^{\prime}\left(\Lambda(p, w, x, \lambda) ;\left(h_{1}, h_{2}, 0,0\right)\right)=\left[\begin{array}{c}
h_{1} \\
h_{2} \\
k_{1} \\
k_{2}
\end{array}\right] \text { as }\left[\begin{array}{c}
h_{1} \\
h_{2} \\
0 \\
0
\end{array}\right]=\Lambda^{\prime}\left((p, w, x, \lambda) ;\left(h_{1}, h_{2}, k_{1}, k_{2}\right)\right) .
$$

Remembering from Lemma 5.1 that for any $(p, w)$ sufficiently close to $(\bar{p}, \bar{w})$ we have

$$
\pi(p, w)=\Pi_{\Gamma(w)}(p)=\left(I+N_{\Gamma(w)}\right)^{-1}(p) .
$$

Shrinking $O_{1} \times O_{2}$ if necessary, find $(x, \lambda) \in O_{5} \times O_{6}$ so that $\sigma(p, w, 0,0)=\left(\sigma_{1}(p, w, 0,0), \sigma_{2}(p, w, 0,0)\right)=$ $(x, \lambda)$ with $(p, w) \in O_{1} \times O_{6}$. It follows from (5.10) that $\pi(p, w)=\sigma_{1}(p, w, 0,0)$ and thus

$$
\begin{aligned}
\pi^{\prime}\left((p, w) ;\left(h_{1}, h_{2}\right)\right) & =\lim _{t \rightarrow 0} \frac{\pi\left(p+t h_{1}, w+t h_{2}\right)-\pi(p, w)}{t} \\
& =\lim _{t \rightarrow 0} \frac{\sigma_{1}\left(p+t h_{1}, w+t h_{2}, 0,0\right)-\sigma_{1}(p, w, 0,0)}{t}=k_{1}
\end{aligned}
$$


where the last equality is a result of the first equation in (5.9). Employing finally the second equation in (5.9), we conclude the proof of the theorem.

To obtain the main result of this section given in Theorem 5.4, we need to establish one more property of the projection mapping $\pi(p, w)$ defined in Lemma 5.1. This is done in the next lemma by using again the obtained second-order characterization of full stability.

Lemma 5.3 (Lipschitz continuity of projections). Suppose that $\Phi(\bar{x}, \bar{w}) \in \Theta$ and that assumptions (RC) and (ND) hold at $(\bar{x}, \bar{w})$. Then the projection mapping $(p, w) \longmapsto \pi(p, w)$ is Lipschitz continuous around $(\bar{p}, \bar{w})$ with $\bar{p}=\bar{x}$.

Proof. Consider the parametric problem $\mathcal{P}(p, w, v)$ in the setting of full stability defined by:

$$
\text { minimize } \frac{1}{2}\|x-p\|^{2}-\langle v, x\rangle \text { over } \Phi(x, w) \in \Theta
$$

where $p, v \in \mathbb{R}^{n}, w \in \mathbb{R}^{d}$. Similar to the proof of Theorem 5.2 we show that $\bar{x}$ is a fully stable local minimizer of $\mathcal{P}(\bar{p}, \bar{w}, 0)$. Hence there is $\gamma>0$ such that the mapping $(p, w, v) \longmapsto M_{\gamma}(p, w, v)$ from (3.5) is single-valued and Lipschitz continuous around $(\bar{p}, \bar{w}, 0)$, where

$$
\varphi(x, p, w):=\frac{1}{2}\|x-p\|^{2} .
$$

Therefore we can find a neighborhood $U \times O \times V$ of $(\bar{p}, \bar{w}, 0)$ on which $M_{\gamma}$ is Lipschitz continuous. Suppose without loss of generality that $U \subset \mathbb{B}_{\varepsilon}(\bar{p}), O \subset W$, and $\mathbb{B}_{\gamma}(\bar{x}) \subset \mathbb{B}_{\nu}(\bar{x})$ with $\varepsilon, \nu$, and $W$ taken from Lemma 5.1(ii). Since $M_{\gamma}(p, w, 0)$ is the optimal solution to (5.11) for any $(p, w) \in U \times O$, it yields $0 \in \xi-p+N_{\Gamma(w)}(\xi)$ with $\xi:=M_{\gamma}(p, w, 0)$. Thus we deduce that

$$
\xi \in\left(I+N_{\Gamma(w)}\right)^{-1}(p) \cap \mathbb{B}_{\gamma}(\bar{x}) \subset\left(I+N_{\Gamma(w)}\right)^{-1}(p) \cap \mathbb{B}_{\nu}(\bar{x})=\pi(p, w) .
$$

Taking into account that $\pi(p, w)$ is single-valued on $U \times O$ gives us $\pi(p, w)=\xi=M_{\gamma}(p, w, 0)$ for any $(p, w) \in U \times O$ and thus justifies the claimed local Lipschitz continuity of $\pi$.

Now we are ready to establish the main result of this section providing a verifiable formula for calculating the graphical derivative (2.7) of the normal cone mapping (5.2) generated by the moving sets (5.1) entirely via the initial data. The obtained result reduces to [28, Theorem 3.3] when the set $\Gamma$ in (5.1) is constant and convex. We also refer the reader to the recent preprint [29], which contains a counterpart of the latter result in the case of constant while nonconvex sets $\Gamma$ satisfying certain requirements, which may not hold for general convex sets.

Theorem 5.4 (calculating graphical derivatives). Let $\bar{v} \in \Xi(\bar{x}, \bar{w})$ in $(5.2)$ with $\bar{x} \in \Gamma(\bar{w})$ under the assumptions of Theorem 5.2, and let $\bar{\lambda} \in \mathbb{R}^{m}$ solve the the KKT system (5.4) associated with $(p, w, x)=(\bar{x}+\bar{v}, \bar{w}, \bar{x})$. Then we have the tangent cone formula

$$
\begin{aligned}
& T_{\mathrm{gph} \Xi}(\bar{x}, \bar{w}, \bar{v})=\left\{(a, b, c) \in \mathbb{R}^{n} \times \mathbb{R}^{d} \times \mathbb{R}^{n} \mid \exists q \in \mathbb{R}^{m}, t \in(0,1)\right. \text { with } \\
& \quad c=\sum_{i=1}^{m} \bar{\lambda}_{i} \nabla_{x x}^{2} \Phi_{i}(\bar{x}, \bar{w}) a+\sum_{i=1}^{m} \bar{\lambda}_{i} \nabla_{x w}^{2} \Phi_{i}(\bar{x}, \bar{w}) b+\frac{1}{t}\left(\nabla_{x} \Phi(\bar{x}, \bar{w})\right)^{*} q, \\
& \left.0=\nabla_{x} \Phi(\bar{x}, \bar{w}) a+\nabla_{w} \Phi(\bar{x}, \bar{w}) b-\Pi_{\Theta}^{\prime}\left(\Phi(\bar{x}, \bar{w})+t \bar{\lambda} ; \nabla_{x} \Phi(\bar{x}, \bar{w}) a+\nabla_{w} \Phi(\bar{x}, \bar{w}) b+q\right)\right\} .
\end{aligned}
$$

Furthermore, for each $a \in \mathbb{R}^{n}$ the graphical derivative of $\Xi$ at $(\bar{x}, \bar{w}, \bar{v})$ is calculated by

$$
\begin{aligned}
& D \Xi(\bar{x}, \bar{w}, \bar{v})(a)=\left\{(b, c) \in \mathbb{R}^{d} \times \mathbb{R}^{n} \mid \exists q \in \mathbb{R}^{m}, t \in(0,1)\right. \text { with } \\
& \quad c=\sum_{i=1}^{m} \bar{\lambda}_{i} \nabla_{x x}^{2} \Phi_{i}(\bar{x}, \bar{w}) a+\sum_{i=1}^{m} \bar{\lambda}_{i} \nabla_{x w}^{2} \Phi_{i}(\bar{x}, \bar{w}) b+\frac{1}{t}\left(\nabla_{x} \Phi(\bar{x}, \bar{w})\right)^{*} q, \\
& \left.\quad 0=\nabla_{x} \Phi(\bar{x}, \bar{w}) a+\nabla_{w} \Phi(\bar{x}, \bar{w}) b-\Pi_{\Theta}^{\prime}\left(\Phi(\bar{x}, \bar{w})+t \bar{\lambda} ; \nabla_{x} \Phi(\bar{x}, \bar{w}) a+\nabla_{w} \Phi(\bar{x}, \bar{w}) b+q\right)\right\} .
\end{aligned}
$$


Proof. Since the nondegeneracy condition (ND) implies the validity of RCQ (3.8), we find $\varepsilon>0$ and $\gamma>0$ such that assertion (ii) of Lemma 5.1 holds. Fixing the neighborhood $O_{1}$ from Theorem 5.2, observe from the proof of that theorem that $O_{1} \subset \mathbb{B}_{\varepsilon}(\bar{p})$ with $\bar{p}=\bar{x}$. Let $t \in(0,1)$ be so small that $\bar{x}+t \bar{v} \in O_{1}$. Denoting $p:=\bar{x}+t \bar{v}$, we get $p \in\left(I+N_{\Gamma(\bar{w})}\right)(\bar{x})$ and $p \in \mathbb{B}_{\varepsilon}(\bar{p})$. Applying now (5.10) tells us that $\pi(p, \bar{w})=\bar{x}$. It follows from the Lipschitz continuity of $\pi$ in Lemma 5.3 and [12, Proposition 3.1] that $T_{\operatorname{gph} \pi}(p, \bar{w}, \bar{x})=\operatorname{gph} \pi^{\prime}((p, \bar{w}) ;(\cdot, \cdot))$. Employing (5.10) again, we arrive at the equality gph $\Xi=F^{-1}(\operatorname{gph} \pi)$ on a neighborhood of $(\bar{x}, \bar{w}, t \bar{v})$ with $F(x, y, z):=(x+z, y, x)$. This gives us by the calculus result from [39, Exercise 6.7] that

$$
\begin{aligned}
T_{\mathrm{gph} \Xi}(\bar{x}, \bar{w}, t \bar{v}) & =\left\{(a, b, c) \in \mathbb{R}^{n} \times \mathbb{R}^{d} \times \mathbb{R}^{n} \mid \nabla F(\bar{x}, \bar{w}, t \bar{v})\left[\begin{array}{l}
a \\
b \\
c
\end{array}\right] \in T_{\operatorname{gph} \pi}(p, \bar{w}, \bar{x})\right\} \\
& =\left\{(a, b, c) \in \mathbb{R}^{n} \times \mathbb{R}^{d} \times \mathbb{R}^{n} \mid \nabla F(\bar{x}, \bar{w}, t \bar{v})\left[\begin{array}{c}
a \\
b \\
c
\end{array}\right] \in \operatorname{gph} \pi^{\prime}((p, \bar{w}) ;(\cdot, \cdot))\right\} \\
& =\left\{(a, b, c) \in \mathbb{R}^{n} \times \mathbb{R}^{d} \times \mathbb{R}^{n} \mid\left[\begin{array}{c}
a+c \\
b \\
c
\end{array}\right] \in \operatorname{gph} \pi^{\prime}((p, \bar{w}) ;(\cdot, \cdot))\right\} .
\end{aligned}
$$

Using these together with Theorem 5.2, we get the expression

$$
\begin{aligned}
& T_{\mathrm{gph} \Xi}(\bar{x}, \bar{w}, t \bar{v})=\left\{(a, b, c) \mid \exists q \in \mathbb{R}^{m}\right. \text { with } \\
& \quad c=t\left(\sum_{i=1}^{m} \bar{\lambda}_{i} \nabla_{x x}^{2} \Phi_{i}(\bar{x}, \bar{w}) a+\sum_{i=1}^{m} \bar{\lambda}_{i} \nabla_{x w}^{2} \Phi_{i}(\bar{x}, \bar{w})\right)+\nabla_{x} \Phi(\bar{x}, \bar{w})^{*} q, \\
& \left.\quad 0=\nabla_{x} \Phi(\bar{x}, \bar{w}) a+\nabla_{w} \Phi(\bar{x}, \bar{w}) b-\Pi_{\Theta}^{\prime}\left(\Phi(\bar{x}, \bar{w})+t \bar{\lambda} ; \nabla_{x} \Phi(\bar{x}, \bar{w}) a+\nabla_{w} \Phi(\bar{x}, \bar{w}) b+q\right)\right\}
\end{aligned}
$$

with $\bar{\lambda}$ from the formulation of the theorem. It gives us by implementing [11, Lemma 3.1] that

$$
(a, b, t c) \in T_{\operatorname{gph} \Xi}(\bar{x}, \bar{w}, t \bar{v}) \Longleftrightarrow(a, b, c) \in T_{\operatorname{gph} \Xi}(\bar{x}, \bar{w}, \bar{v}),
$$

which justifies together with the previous formula the representation of the tangent cone claimed in the theorem. The latter directly yields the asserted graphical derivative expression by definition (2.7) and thus completes the proof of the theorem.

\section{Full Stability of Solutions to Minimax Problems}

This section is mainly devoted to the study of full stability for the following minimax problem:

$$
\text { minimize } \psi(x):=\max \left\{\varphi_{1}(x), \ldots, \varphi_{m}(x)\right\} \text { over } x \in \mathbb{R}^{n},
$$

where $\varphi_{i}: \mathbb{R}^{n} \rightarrow \mathbb{R}, i=1, \ldots, m$, are $\mathcal{C}^{2}$-smooth around the reference points. We also consider some related topics of variational analysis and second-order generalized differentiation, which are of their own interest while being important for our study of minimax problems.

The class of intrinsically nonsmooth maximum functions $\psi$ in (6.1) has been well recognized in nonlinear analysis, optimization, and their various applications. As mentioned in [39, Example 10.24(e)], it belongs to a broader class of "nice" nonsmooth functions defined as follows [39, Definition 10.23]: $\varphi: \mathbb{R}^{n} \rightarrow \overline{\mathbb{R}}$ is fully amenable at $\bar{x} \in \operatorname{dom} \varphi$ if there is an neighborhood $V$ of $\bar{x}$ on which $\varphi=\theta \circ \Phi$ for a $\mathcal{C}^{2}$-smooth mapping $\Phi: V \rightarrow \mathbb{R}^{m}$ and a proper 1.s.c. and convex piecewise linear-quadratic function $\theta: \mathbb{R}^{m} \rightarrow \overline{\mathbb{R}}$ such that the qualification condition

$$
\partial^{\infty} \theta(\Phi(\bar{x})) \cap \operatorname{ker} \nabla \Phi(\bar{x})^{*}=\{0\}
$$


is satisfied. Indeed, observe that in the case of (6.1) we have the composition

$$
\psi(x)=(\theta \circ \Phi)(x), \quad x \in \mathbb{R}^{n},
$$

with $\Phi(x):=\left(\varphi_{1}(x), \ldots, \varphi_{m}(x)\right)$ and the component maximum function $\theta: \mathbb{R}^{m} \rightarrow \mathbb{R}$ defined by

$$
\theta(z):=\max \left\{z_{1}, \ldots, z_{m}\right\} \text { for } z=\left(z_{1}, \ldots, z_{m}\right) \in \mathbb{R}^{m},
$$

where the qualification condition (6.2) holds whenever $\bar{x} \in \mathbb{R}^{n}$ due to the Lipschitz continuity of (6.4) on $\mathbb{R}^{n}$. Note furthermore that the convex function $\theta$ in (6.4) is in fact piecewise linear, i.e., its epigraph is a polyhedral subset of $\mathbb{R}^{m+1}$. This means that the maximum function $\psi$ in $(6.1)$ belongs to a remarkable subclass of fully amenable functions corresponding to piecewise linear outer functions in compositions (6.3). It is also worth mentioning the useful representation of the component maximum function (6.4) in the form

$$
\theta\left(z_{1}, \ldots, z_{m}\right)=\sigma_{\mathcal{M}}(z), \quad z \in \mathbb{R}^{m}
$$

where $\sigma_{\mathcal{M}}(z):=\max \{\langle z, u\rangle \mid u \in \mathcal{M}\}$ stands for the support function of the unit simplex $\mathcal{M}:=\left\{u=\left(u_{1}, \ldots, u_{m}\right) \mid u_{i} \geq 0, \sum_{i=1}^{m} u_{i}=1\right\}$ in $\mathbb{R}^{m}$.

The main result of this section given in Theorem 6.2 provides a complete characterization of full stability of local minimizers for (6.1) expressed entirely in terms of the initial data of this problem. After some preparation, we provide two alternative proofs of the main result. The first one is based on reducing the minimax problem under consideration to an extended nonlinear program in the sense of [38] with applying a characterization of full stability for such problems derived in [32]. The other proof involves a characterization of full stability in composite optimization problems with general piecewise linear functions $\theta$ in amenable compositions (6.3) given in [32] and then applying direct calculations of the second-order subdifferential of $\theta$ in (6.4) obtained in [9] and related to Proposition 6.1 presented below.

To proceed, take a subgradient $\bar{v} \in \partial \theta(\bar{z})$ of the function $\theta$ in $(6.4)$ with $\bar{v}=\left(\bar{v}_{1}, \ldots, \bar{v}_{m}\right) \in \mathbb{R}^{m}$ and $\bar{z}=\left(\bar{z}_{1}, \ldots, \bar{z}_{m}\right) \in \mathbb{R}^{m}$. We obviously have $\bar{v}_{i} \geq 0, i=1, \ldots, m$. Considering the index sets

$$
\begin{gathered}
I_{+}(\bar{v}):=\left\{i \in\{1, \ldots, m\} \mid \bar{v}_{i}>0\right\}, \quad I_{0}(\bar{v}):=\left\{i \in\{1, \ldots, m\} \mid \bar{v}_{i}=0\right\}, \\
J_{a}(\bar{z}):=\left\{i \in\{1, \ldots, m\} \mid \bar{z}_{i}=\theta(\bar{z})\right\}, \quad \text { and } \quad J_{n a}(\bar{z}):=\left\{i \in\{1, \ldots, m\} \mid \bar{z}_{i}<\theta(\bar{z})\right\},
\end{gathered}
$$

it is easy to check the well-known "complementarity" inclusion $J_{n a}(\bar{z}) \subset I_{0}(\bar{v})$.

The next proposition calculates the second-order subdifferential of $\theta$ via closed faces of the corresponding critical cone. Recall that a closed face of the given closed cone $K \subset \mathbb{R}^{m}$ is

$$
C:=\{z \in K \mid\langle z, y\rangle=0\} \text { for some } y \in K^{*}
$$

via the dual cone $K^{*}:=\left\{y \in \mathbb{R}^{m} \mid\langle y, z\rangle \leq 0\right.$ for all $\left.z \in K\right\}$.

Proposition 6.1 (second-order subdifferential of the component maximum function). Let $\bar{v} \in \partial \theta(\bar{z})$ for $\theta$ in (6.4). Then the second-order subdifferential of $\theta$ at $(\bar{z}, \bar{v})$ is calculated by

$$
q \in \partial^{2} \theta(\bar{z}, \bar{v})(p) \Longleftrightarrow\left\{\begin{array}{l}
\text { there exist closed faces } K_{1} \subset K_{2} \text { of } K \\
\text { with }-q \in K_{1}-K_{2},-p \in\left(K_{2}-K_{1}\right)^{*},
\end{array}\right.
$$

where $K:=T_{\mathcal{M}}(\bar{v}) \cap \bar{z}^{\perp}$ is the critical cone of the unit simplex $\mathcal{M}$ at $(\bar{z}, \bar{v})$ expressed as

$$
\begin{aligned}
& K=\left\{\left(w_{1}, \ldots, w_{m}\right) \mid \sum_{i \in J_{a}(\bar{z})} w_{i}=0, \quad w_{i} \geq 0 \text { for all } i \in I_{0}(\bar{v}) \cap J_{a}(\bar{z}),\right. \\
& \left.w_{i}=0 \text { for all } i \in J_{n a}(\bar{z})\right\} \text {. }
\end{aligned}
$$


Proof. We get from representation (6.5) and the conjugacy relation $\sigma_{\mathcal{M}}=\delta_{\mathcal{M}}^{*}$ that

$$
q \in \partial^{2} \theta(\bar{z}, \bar{v})(p) \Longleftrightarrow-p \in \partial^{2} \delta_{\mathcal{M}}(\bar{v}, \bar{z})(-q) .
$$

This allows us to conclude from the proof of [4, Theorem 2] that

$$
-p \in \partial^{2} \delta_{\mathcal{M}}(\bar{v}, \bar{z})(-q) \Longleftrightarrow\left\{\begin{array}{l}
\text { there exist closed faces } K_{1} \subset K_{2} \text { of } K \\
\text { such that }-q \in K_{1}-K_{2},-p \in\left(K_{2}-K_{1}\right)^{*}
\end{array}\right.
$$

via the critical cone $K=T_{\mathcal{M}}(\bar{v}) \cap \bar{z}^{\perp}$, which gives us (6.8). It remains to verify representation (6.9). We can easily deduce from the normal cone definition that

$$
N_{\mathcal{M}}(\bar{v})=\left\{\left(\alpha_{1}, \ldots, \alpha_{m}\right) \mid \alpha_{i_{1}}=a_{i_{2}}=: \xi \text { for } i_{1}, i_{2} \in I_{+}(\bar{v}), \xi \geq \alpha_{i} \text { for } i \in I_{0}(\bar{v})\right\},
$$

and thus the tangent cone to $\mathcal{M}$ is expressed by duality as

$$
T_{\mathcal{M}}(\bar{v})=\left(N_{\mathcal{M}}(\bar{v})\right)^{*}=\left\{\left(w_{1}, \ldots, w_{m}\right) \mid \sum_{i=1}^{m} w_{i}=0, w_{i} \geq 0 \text { for } i \in I_{0}(\bar{v})\right\} .
$$

Pick now $w \in K$ and get by constructions in (6.7) that

$$
\sum_{i \in J_{a}(\bar{z})} w_{i} \theta(\bar{z})+\sum_{i \in J_{n a}(\bar{z})} w_{i} \bar{z}_{i}=0 .
$$

Taking into account that $\sum_{i=1}^{m} w_{i}=0$ due to $w \in T_{\mathcal{M}}(\bar{v})$, we have

$$
\sum_{i \in J_{n a}(\bar{z})} w_{i}\left(\bar{z}_{i}-\theta(\bar{z})\right)=0
$$

and then arrive by the inclusion $J_{n a}(\bar{z}) \subset I_{0}(\bar{v})$ at $w_{i} \geq 0$ for $i \in J_{n a}(\bar{z})$. Using this together with (6.10) tells us that $w_{i}\left(\bar{z}_{i}-\theta(\bar{z})\right)=0$ for all $i \in J_{n a}(\bar{z})$, which implies in turn that $w_{i}=0$ for these indices. Hence $w$ belongs to the set on the right-hand side of (6.9). The converse inclusion therein is verified similarly, and thus we complete the proof of the proposition.

Now we are ready to proceed with full stability analysis of the minimax problem (6.1). Consider the corresponding full perturbation problem $\mathcal{P}(w, v)$ given as follows:

$$
\text { minimize } \psi(x, w)-\langle x, v\rangle \text { over } x \in \mathbb{R}^{n}
$$

with $(w, v) \in \mathbb{R}^{d} \times \mathbb{R}^{n}$ and $\psi$ defined in (6.3). The next theorem provides a complete characterization of full stability for the minimax problem under consideration entirely in terms of its initial data. We present two alternatives proofs of this major result.

Theorem 6.2 (characterizing full stability of locally optimal solutions to minimax problems). Let $(\bar{x}, \bar{w}) \in \mathbb{R}^{n} \times \mathbb{R}^{d}$ for problem $\mathcal{P}(\bar{w}, \bar{v})$ in (6.11) with $\bar{v} \in \partial_{x} \psi(\bar{x}, \bar{w})$, where $\psi=\theta \circ \Phi$ with $\Phi=\left(\varphi_{1}, \ldots, \varphi_{m}\right)$ and the component maximum function $\theta$ is taken from (6.4). Let $\bar{p} \in \partial \theta(\bar{z})$ with $\bar{z}=\Phi(\bar{x})$ be the unique vector satisfying the conditions $\nabla_{x} \Phi(\bar{x}, \bar{w})^{*} \bar{p}=\bar{v}$ and

$$
(K-K) \cap \operatorname{ker} \nabla_{x} \Phi(\bar{x}, \bar{w})^{*}=\{0\},
$$

where $K=K(\bar{z}, \bar{v})$ is the critical cone (6.9) with $K-K$ calculated by

$$
K-K=\left\{\left(w_{1}, \ldots, w_{m}\right) \mid \sum_{i \in J_{a}(\bar{z})} w_{i}=0, w_{j}=0 \text { for } j \in J_{n a}(\bar{z})\right\}
$$


via the index sets $J_{a}(\bar{z})$ and $J_{n a}(\bar{z})$ from $(6.7)$. Then $\bar{x}$ is a fully stable local minimizer of $\mathcal{P}(\bar{w}, \bar{v})$ if and only if the Hessian matrix $\nabla_{x x}^{2} \Phi(\bar{x}, \bar{w})^{*} \bar{p}$ is positive definite on the subspace

$$
\mathcal{N}:=\left\{u \in \mathbb{R}^{n} \mid \nabla_{x} \varphi_{i}(\bar{x}, \bar{w}) u=c \text { for all } i \in I_{+}(\bar{p})\right\},
$$

where the constant $c \in \mathbb{R}$ is arbitrary, and where $I_{+}(\bar{p})$ is the index set defined in (6.6).

Proof. Observe first that the original minimax problem (6.1) can be written in the following form of extended nonlinear programming (ENLP) introduced by Rockafellar [38]:

$$
\left\{\begin{array}{l}
\operatorname{minimize} \varphi_{0}(x)+(\theta \circ \Phi)(x) \text { over } x \in \mathbb{R}^{n} \\
\text { with } \theta(z):=\sup _{p \in P}\{\langle p, z\rangle-\vartheta(p)\}
\end{array}\right.
$$

where the functions $\varphi_{0}: \mathbb{R}^{n} \rightarrow \mathbb{R}$ and $\Phi: \mathbb{R}^{n} \rightarrow \mathbb{R}^{m}$ are smooth while the function $\vartheta: \mathbb{R}^{m} \rightarrow \mathbb{R}$ is smooth and convex on the nonempty polyhedral set

$$
P:=\left\{p \in \mathbb{R}^{m} \mid\left\langle a_{j}, p\right\rangle \leq b_{j} \text { for all } j=1, \ldots, l\right\}
$$

defined by vectors $a_{j} \in \mathbb{R}^{m}$ and numbers $b_{j} \in \mathbb{R}$ for some $l \in \mathbb{N}$. The case of (6.1) corresponds to (6.14) with $\varphi_{0}=0, \Phi=\left(\varphi_{1}, \ldots, \varphi_{m}\right), \vartheta=0$, and $P=\mathcal{M}$ for the unit simplex $\mathcal{M} \subset \mathbb{R}^{m}$ due to representation (6.5). In framework (6.14), consider the extended Lagrangian

$$
\mathcal{L}(x, w, p):=\varphi_{0}(x, w)+\Phi(x, w)^{*} p-\vartheta(p) \text { with } p \in \mathbb{R}^{m},
$$

where $p \in \mathbb{R}^{m}$ signifies a vector of Lagrange multipliers.

We intend to study the full stability of local solutions to the minimax problem by applying [32, Theorem 7.3] that gives a characterization of fully stable minimizers in ENLPs expressed via a certain positive definiteness of the partial Hessian of (6.15). To proceed, we observe first that the second-order qualification condition

$$
\partial^{2} \theta(\bar{z}, \bar{p})(0) \cap \operatorname{ker} \nabla_{x} \Phi(\bar{x}, \bar{w})^{*}=\{0\}
$$

imposed in [32, Theorem 7.3] is equivalent to (6.12) in our case. Indeed, it follows from calculating the second-order subdifferential of $\theta$ in Propositions 6.1 that $\partial^{2} \theta(\bar{z}, \bar{p})(0)=K-K$, which thus verifies the claimed equivalence. Note also that, although the result of [32, Theorem 7.3] is formulated under the full rank condition on $\nabla_{x} \Phi(\bar{x}, \bar{w})$, the latter is required for the general case of ENLPs considered therein. As follows from the proof of [32, Theorem 7.3], in the case of piecewise linear functions $\theta$ in (6.14), which includes (6.4), the result of that theorem holds with the replacement of the full rank condition by the weaker nondegeneracy one (6.12).

Having this in mind, we are now ready to apply the characterization of full stability of local minimizers for ENLPs (6.14) obtained in [32, Theorem 7.3] via the following extended strong second-order optimality condition (ESSOC). Given $(\bar{x}, \bar{w}, \bar{v}, \bar{p})$ with $\nabla_{x} \mathcal{L}(\bar{x}, \bar{w} \bar{p})=\bar{v}$, the ESSOC means that the conditional positive definiteness of the Hessian

$$
\left\langle u, \nabla_{x x}^{2} \mathcal{L}(\bar{x}, \bar{w}, \bar{p}) u\right\rangle>0 \text { for all } 0 \neq u \in \mathcal{S}
$$

on the subspace $\mathcal{S} \subset \mathbb{R}^{n}$ defined by

$$
\mathcal{S}:=\left\{u \in \mathbb{R}^{n} \mid \nabla_{x} \Phi(\bar{x}, \bar{w}) u \in \operatorname{span}\left\{a_{j} \mid j \in I(\bar{p})\right\}\right\}
$$

with the active constraint index set $I(\bar{p})=\left\{j \in\{1, \ldots, l\} \mid\left\langle a_{j}, \bar{p}\right\rangle=b_{j}\right\}$ at $\bar{p} \in P$. 
It remains to expressed conditions (6.17) and (6.18) in terms of the function $\theta$ from (6.5). In this setting we have $\mathcal{L}(x, w, p):=\Phi(x, w)^{*} p$ and thus need to verify that $\mathcal{N}$ in $(6.13)$ is a subspace reducing to $\mathcal{S}$ in (6.18). Since $P=\mathcal{M}$ for the unit simplex $\mathcal{M} \subset \mathbb{R}^{m}$ in (6.5), it follows that $a_{i}=-e_{i}$ for $i=1, \ldots, m$, where each $e_{i} \in \mathbb{R}^{m}$ is the unit vector such that the $\mathrm{j}^{\mathrm{th}}$ component of it is 1 while the others are 0 , and where $a_{m+1}$ is the vector in $\mathbb{R}^{m}$ for which all the components are equal to 1 . Thus we have the representation

$$
P=\left\{p \in \mathbb{R}^{m} \mid\left\langle-e_{j}, p\right\rangle \leq 0, \text { for } j=1, \ldots, m \text { and }\left\langle a_{m+1}, p\right\rangle=1\right\} .
$$

Observe that $I(\bar{p})=I_{0}(\bar{p}) \cup\{m+1\}$ with $I_{0}(\bar{p})$ is given in (6.6). To show that $\mathcal{S}=\mathcal{N}$, pick $u \in \mathcal{S}$ and find $\alpha_{i} \in \mathbb{R}$ for $i \in I(\bar{p})$ satisfying

$$
\nabla_{x} \Phi(\bar{x}, \bar{w}) u=\sum_{i \in I(\bar{p})} \alpha_{i} a_{i}=\sum_{i \in I_{0}(\bar{p})} \alpha_{i} e_{i}+\alpha_{m+1} a_{m+1}
$$

which in turn implies that $\nabla_{x} \varphi_{i}(\bar{x}, \bar{w}) u=\alpha_{m+1}$ whenever $i \in I_{+}(\bar{p})$, and hence we get $u \in \mathcal{N}$. Conversely, take $u \in \mathcal{N}$ and find a constant $c \in \mathbb{R}$ such that $\nabla_{x} \varphi_{i}(\bar{x}, \bar{w}) u=c$ for all $i \in I_{+}(\bar{p})$. Letting $\alpha_{i}:=\nabla_{x} \varphi_{i}(\bar{x}, \bar{w}) u-c$ for $i \in I_{0}(\bar{p})$ and $\alpha_{m+1}:=c$ leads us to equality (6.19), which shows that $u \in \mathcal{S}$ and thus completes the proof of the theorem.

Alternative proof of Theorem 6.2. Consider the more general setting of Theorem 6.2, where the outer function $\theta: \mathbb{R}^{m} \rightarrow \overline{\mathbb{R}}$ in the composition $\theta \circ \Phi$ is arbitrary piecewise linear under the validity of the second-order qualification condition (6.16), which reduces to (6.12) for $\theta$ from (6.4). It follows from [32, Theorem 5.2(ii)] that the full stability of $\bar{x}$ in $\mathcal{P}(\bar{w}, \bar{v})$ in the notation above is characterized by the second-order condition

$$
\left\langle\nabla_{x x}^{2} \Phi(\bar{x}, \bar{w})(u, u), \bar{p}\right\rangle+\left\langle q, \nabla_{x} \Phi(\bar{x}, \bar{w}) u\right\rangle>0 \text { for all } q \in \partial^{2} \theta(\bar{z}, \bar{p})\left(\nabla_{x} \Phi(\bar{x}, \bar{w}) u\right)
$$

with $u \neq 0$. Employing further the results of [9, Theorem 3.1], we deduce that

$$
\operatorname{dom} \partial^{2} \theta(\bar{z}, \bar{p})=\left\{u=\left(u_{1}, \ldots, u_{n}\right) \in \mathbb{R}^{n} \mid u_{i}=c \text { whenever } i \in I_{+}(\bar{p})\right\}
$$

for $\theta$ in (6.4) with some constant $c \in \mathbb{R}$ and $I_{+}(\bar{p}):=\left\{i \in\{1, \cdots, m\} \mid \bar{p}_{i}>0\right\}$. Moreover, it follows from $[9$, Theorem 3.1] that

$$
\begin{aligned}
\partial^{2} \theta(\bar{z}, \bar{p})\left(\nabla_{x} \Phi(\bar{x}, \bar{w}) u\right)=\left\{q=\left(q_{1}, \ldots, q_{m}\right) \in \mathbb{R}^{m} \mid\right. & \sum_{i=1}^{m} q_{i}=0, q_{i} \geq 0 \text { for } j \in J_{>}, \\
& \left.q_{i}=0 \text { for } j \in J_{n a}(\bar{z}) \cup J_{<}\right\}
\end{aligned}
$$

provided that $\nabla_{x} \Phi(\bar{x}, \bar{w}) u \in \operatorname{dom} \partial^{2} \theta(\bar{z}, \bar{p})$, where $J_{>}:=\left\{i \in J_{a}(\bar{z}) \mid \nabla_{x} \varphi_{i}(\bar{x}, \bar{w}) u>c\right\}$ and $J_{<}:=\left\{i \in J_{a}(\bar{z}) \mid \nabla_{x} \varphi_{i}(\bar{x}, \bar{w}) u<c\right\}$. Now a simple observation reveals that

$$
\nabla_{x} \Phi(\bar{x}, \bar{w}) u \in \operatorname{dom} \partial^{2} \theta(\bar{z}, \bar{p}) \Longleftrightarrow u \in \mathcal{N}
$$

with $\mathcal{N}$ from (6.13). Substituting this into (6.20) and letting $q=0$ gives us

$$
\left\langle\nabla_{x x}^{2} \Phi(\bar{x}, \bar{w})(u, u), \bar{p}\right\rangle>0 \text { whenever } u \in \mathcal{N}, u \neq 0,
$$

and thus we arrive at the positive definiteness of $\nabla_{x x}^{2} \Phi(\bar{x}, \bar{w})^{*} \bar{p}$ on the subspace $\mathcal{N}$. 
Conversely, suppose that the latter condition holds and pick $q \in \partial^{2} \theta(\bar{z}, \bar{p})\left(\nabla_{x} \Phi(\bar{x}, \bar{w}) u\right)$ with $u \neq 0$. To show that $\bar{x}$ is a fully stable local minimizer of $\mathcal{P}(\bar{w}, \bar{v})$ in $(6.11)$, it suffices to verify that $\left\langle q, \nabla_{x} \Phi(\bar{x}, \bar{w}) u\right\rangle \geq 0$. Taking into account that $\nabla_{x} \Phi(\bar{x}, \bar{w}) u \in \operatorname{dom} \partial^{2} \theta(\bar{z}, \bar{p})$, we get

$$
\begin{aligned}
\left\langle q, \nabla_{x} \Phi(\bar{x}, \bar{w}) u\right\rangle & =\sum_{i=1}^{m} q_{i} \nabla_{x} \varphi_{i}(\bar{x}, \bar{w}) u=\sum_{i \in J_{n a}(\bar{z})} q_{i} \nabla_{x} \varphi_{i}(\bar{x}, \bar{w}) u+\sum_{i \in J_{<}} q_{i} \nabla_{x} \varphi_{i}(\bar{x}, \bar{w}) u \\
& +\sum_{i \in J_{>}} q_{i} \nabla_{x} \varphi_{i}(\bar{x}, \bar{w}) u+\sum_{i \in J_{=}} q_{i} \nabla_{x} \varphi_{i}(\bar{x}, \bar{w}) u \\
& =\sum_{i \in J_{>}} q_{i} \nabla_{x} \varphi_{i}(\bar{x}, \bar{w}) u-c \sum_{i \in J_{>}} q_{i}=\sum_{i \in J_{>}} q_{i}\left(\nabla_{x} \varphi_{i}(\bar{x}, \bar{w}) u-c\right) \geq 0
\end{aligned}
$$

with $J_{=}:=\left\{i \in J_{a}(\bar{z}) \mid \nabla_{x} \varphi_{i}(\bar{x}, \bar{w}) u=c\right\}$. Using this together with the assumed positive definiteness of $\nabla_{x x}^{2} \Phi(\bar{x}, \bar{w})^{*} \bar{p}$ on the subspace $\mathcal{N}$ tells us that (6.20) holds and thus justifies that $\bar{x}$ is a fully stable local minimizer of $\mathcal{P}(\bar{w}, \bar{v})$ in $(6.11)$.

Acknowledgement. The authors thank Francisco Facchinei for drawing our attention to the importance of studying full stability of minimax problems and second-order generalized differentiability properties of maximum functions from the viewpoint of numerical optimization. We also grateful to René Henrion for his helpful remarks on the original version of Section 6 and sharing with us his impressive results from the paper with Konstantin Emich [9]. Useful remarks by anonymous referees and Tran Nghia allowed us to improve the original presentation.

\section{References}

[1] J. F. Bonnans, R. Cominetti and A. Shapiro, Sensitivity analysis of optimization problems under second order regular constraints, Math. Oper. Res. 23 (1998), 806-831.

[2] J. F. Bonnans and A. Shapiro, Perturbation Analysis of Optimization Problems, Springer, New York, 2000.

[3] C. Ding, D. Sun and J. J. Ye, First order optimality conditions for mathematical programs with semidefinite cone complementarity constraints, Math. Program. 147, 539-579.

[4] A. L. Dontchev and R. T. Rockafellar, Characterizations of strong regularity for variational inequalities over polyhedral convex sets, SIAM J. Optim. 6 (1996), 1087-1105.

[5] A. L. Dontchev and R. T. Rockafellar, Implicit Functions and Solution Mappings: A View from Variational Analysis, Springer, Dordrecht, 2009.

[6] D. Drusvyatskiy and A. S. Lewis, Tilt stability, uniform quadratic growth, and strong metric regularity of the subdifferential, SIAM J. Optim. 23 (2013), 256-267.

[7] D. Drusvyatskiy, B. S. Mordukhovich and T. T. A. Nghia, Second-order growth, tilt stability, and metric regularity of the subdifferential, J. Convex Anal. 21 (2014), No. 4.

[8] A. C. Eberhard and R. Wenczel, A study of tilt-stable optimality and sufficient conditions, Nonlinear Anal. 75 (2012), 1260-1281.

[9] K. Emich and R. Henrion, A simple formula for the second-order subdifferential of maximum functions, Vietnam J. Math., DOI 10.1007/s10013-013-0052-0, to appear (2014).

[10] W. L. Hare and R. A. Poliguin, Prox-regularity and stability of the proximal mapping, J. Convex Anal. 14 (2007), 589-606.

[11] R. Henrion, A. Y. Kruger and J. V. Outrata, Some remarks on stability of generalized equations, $J$. Optim. Theory Appl. 159 (2013), 681-697.

[12] R. Henrion, J. V. Outrata and T. Surowiec, On regular coderivatives in parametric equilibria with non-unique multipliers, Math. Program. 136 (2012), 111-131. 
[13] P. Kenderov, Semi-continuity of set-valued monotone mappings, Fund. Math. 88 (1975), 61-69.

[14] D. Klatte and B. Kummer, Nonsmooth Equations in Optimization, Kluwer, Dordrecht, 2002.

[15] M. Kojima, Strongly stable stationary solutions in nonlinear programming, in Analysis and Computation of Fixed Points (S. M. Robinson, ed.), pp. 93-138, Academic Press, New York, 1980.

[16] B. Kummer, Newton's method based on generalized derivatives for nonsmooth functions: Convergence analysis, in: Advances in Optimization (W. Oettli and D. Pallaschke, eds.), pp. 171-194, Lecture Notes Econ. Math. Sci. 382, Springer, Berlin, 1992.

[17] A. B. Levy, R. A. Poliquin and R. T. Rockafellar, Stability of locally optimal solutions, SIAM J. Optim. 10 (2000), 580-604.

[18] A. S. Lewis and S. Zhang, Partial smoothness, tilt stability, and generalized Hessians, SIAM J. Optim. 23 (2013), 74-94.

[19] S. Lu, Implications of the constant rank constraint qualification, Math. Program. 126 (2011), 365392.

[20] B. S. Mordukhovich, Metric approximations and necessary optimality conditions for general classes of extremal problems, Soviet Math. Dokl. 22 (1980), 526-530.

[21] B. S. Mordukhovich, Sensitivity analysis in nonsmooth optimization, in Theoretical Aspects of Industrial Design (D. A. Field and V. Komkov, eds.), pp. 32-46, Proceed. Applied Math. 58, SIAM, Philadelphia, 1992.

[22] B. S. Mordukhovich, Variational Analysis and Generalized Differentiation, I: Basic Theory; II: Applications, Springer, Berlin, 2006.

[23] B. S. Mordukhovich and T. T. A. Nghia, Second-order variational analysis and characterizations of tilt-stable optimal solutions in infinite-dimensional spaces, Nonlinear Anal. 86 (2013), 159-180.

[24] B. S. Mordukhovich and T. T. A. Nghia, Second-order characterizations of tilt stability with applications to nonlinear programming, Math. Program., DOI 10.1007/s10107-014-0806-9, to appear (2014).

[25] B. S. Mordukhovich and T. T. A. Nghia, Full Lipschitzian and Hölderian stability in optimization with applications to mathematical programming and optimal control, SIAM J. Optim. 24 (2014), $1344-1381$.

[26] B. Mordukhovich, T. T. A. Nghia and R. T. Rockafellar, Full stability in finite-dimensional optimization, Math. Oper. Res., DOI 10.1287/moor.2014.0669, to appear (2014).

[27] B. S. Mordukhovich and J. V. Outrata, Tilt stability in nonlinear programming under MangasarianFromovitz constraint qualification, Kybernetika 49 (2013), 446-464.

[28] B. S. Mordukhovich, J. V. Outrata and H. Ramírez C., Second-order variational analysis in conic programming with applications to optimality and stability, preprint (2013); http://www.optimizationonline.org/DB_HTML/2013/01/3723.html.

[29] B. S. Mordukhovich, J. V. Outrata and H. Ramírez C., Graphical derivatives and stability analysis for parameterized equilibria with conic constraints, preprint (2014).

[30] B. S. Mordukhovich, J. V. Outrata and M. E. Sarabi, Full stability of locally optimal solution in second-order cone programming, SIAM J. Optim., DOI 10.1137/130928637, to appear (2014).

[31] B. S. Mordukhovich and R. T. Rockafellar, Second-order subdifferential calculus with application to tilt stability in optimization, SIAM J. Optim. 22 (2012), 953-986.

[32] B. S. Mordukhovich, R. T. Rockafellar and M. E. Sarabi, Characterizations of full stability in constrained optimization, SIAM J. Optim. 23 (2013), 1810-1849.

[33] J. V. Outrata, M. Kočvara and J. Zowe, Nonsmooth Approach to Optimization Problems with Equilibrium Constraints, Kluwer, Dordrecht, 1998. 
[34] J. V. Outrata and H. Ramírez C., On the Aubin property of critical points to perturbed second-order cone programs, SIAM J. Optim. 21 (2011), 798-823.

[35] R. A. Poliquin and R. T. Rockafellar, Tilt stability of a local minimum, SIAM J. Optim. 8 (1998), 287-299.

[36] S. M. Robinson, Strongly regular generalized equations, Math. Oper. Res. 5 (1980), 43-62.

[37] S. M. Robinson, Aspects of the projector on prox-regular sets, in: Variational Analysis and Applications (F. Giannessi and A. Maugeri, eds.), pp. 963-973, Nonconvex Optim. Appl. 79, Springer, New York, 2005.

[38] R. T. Rockafellar, Extended nonlinear programming, in: Nonlinear Optimization and Related Topics (G. Di Pillo and F. Giannessi. eds.), pp. 381-399, Applied Optimization 36, Kluwer Academic Publishers, Dordrecht, 2000,

[39] R. T. Rockafellar and R. J-B. Wets, Variational Analysis, Springer, Berlin, 2006.

[40] M. Sebbah and L. Thibault, Metric projection and compatibly parameterized families of prox-regular sets in Hilbert spaces, Nonlinear Anal. 75 (2012), 1547-1562.

[41] A. Shapiro, Differentiability properties of meyric projections onto convex sets, http://www.optimization-online.org/DB_FILE/2013/11/4119.pdf 